International Journal of Bifurcation and Chaos, Vol. 12, No. 1 (2002) 23-41

(c) World Scientific Publishing Company

\title{
FAMILIES OF SCROLL GRID ATTRACTORS
}

\author{
MÜŞTAK E. YALÇIN*, JOHAN A. K. SUYKENS ${ }^{\dagger}$ and JOOS VANDEWALLE ${ }^{\ddagger}$ \\ Katholieke Universiteit Leuven, \\ Department of Electrical Engineering ESAT-SISTA, \\ Kardinaal Mercierlaan 94, B-3001 Leuven, Belgium \\ *mey@esat.kuleuven.ac.be \\ †johan.suykens@esat.kuleuven.ac.be \\ ${ }_{\ddagger}^{\ddagger}$ joos.vandewalle@esat.kuleuven.ac.be \\ SERDAR ÖZOĞUZ \\ Istanbul Technical University, \\ Faculty of Electrical-Electronics Engineering \\ 80626, Maslak, Istanbul, Turkey \\ serdar@ehb.itu.edu.tr
}

Received February 13, 2001; Revised April 9, 2001

\begin{abstract}
In this paper a new family of scroll grid attractors is presented. These families are classified into three called 1D-, 2D- and 3D-grid scroll attractors depending on the location of the equilibrium points in state space. The scrolls generated from 1D-, 2D- and 3D-grid scroll attractors are located around the equilibrium points on a line, on a plane or in $3 \mathrm{D}$, respectively. Due to the generalization of the nonlinear characteristics, it is possible to increase the number of scrolls in all state variable directions. A number of strange attractors from the scroll grid attractor families are presented. They have been experimentally verified using current feedback opamps. Also Lur'e representations are given for the scroll grid attractor families.
\end{abstract}

\section{Introduction}

Since the discovery of Chua's circuit [Chua et al., 1986; Madan, 1993; Chua, 1994] many scientists from different disciplines have been studying the double scroll family. Chua's circuit is a simple third-order piecewise-linear (PWL) system which has become a paradigm for chaos. The realization of chaotic systems brought chaotic signals into engineering applications. Presently, many researchers investigate the applications of chaotic signals to communication systems [Kolumban et al., 1998; Hasler, 1994]. One open question is how one can systematically increase the complexity of behavior while keeping the systems as simple as possible. The new circuits presented in this paper give an affirmative answer to that question. Amongst the many generalizations of Chua's circuit, a more complicated double scroll family of so-called $n$-double scroll attractors has been proposed by Suykens and Vandewalle [1993] by introducing additional breakpoints in the nonlinearity. A more complete family of $n$-scroll instead of $n$-double scroll attractors has been obtained from a generalized Chua's circuit reported in [Suykens et al., 1997]. Experimental confirmations of 2-double scroll and 5-scroll attractors have been given in [Arena et al., 1996] and [Yalçın et al., 2000a], respectively. The basic idea of generalizing the chaos generators with PWL nonlinearities is to introduce additional breakpoints in the nonlinearity. These breakpoints create equilibrium points which are located on a line in state space. Here, we will consider a new chaos generator which has a simple circuit implementation. The

\footnotetext{
${ }^{\dagger}$ Author for correspondence.
} 
strange attractor families generated from the new chaos generators will be called scroll grid attractors. For these families it is possible to cover the whole state space with scrolls. The new attractor families are classified into three subfamilies according to the location of the equilibrium points:

- 1D-grid scroll attractor family: This is also known as $n$-scroll attractors [Suykens et al., 1997]. The equilibrium points of this family are located on a line and the scrolls generated from the generalized nonlinearity are located around that line along the $x$ state variable direction in state space. Furthermore, the $x$ state variable is also the variable on which the nonlinearity operates.

- 2D-grid scroll attractor family: In this family, the system consists of two nonlinear functions operating on the $x$ and $y$ state variables. The equilibrium points are located in the $x-y$ plane. The scrolls generated from the generalized nonlinearities can be increased in the $x$ and $y$ state variable directions.

- 3D-grid scroll attractor family: This is the most complete class of the presented scroll grid attractor families. The equilibrium points are located in $3 \mathrm{D}$ and the system has three nonlinear functions. Due to the generalization of each nonlinearity, the scrolls can be generated in all state variable directions.

In this paper, the main contribution is to show the possibility of generating the equilibrium points on a plane or in 3D instead of on a line. As a result, it is possible to increase the number of scrolls into all state variable directions. In the literature, a quad screw attractor [Kataoka \& Saito, 2000] from a 4D chaotic oscillator with hysteresis [Saito, 1990] is comparable with a $2 \times 2$-scroll grid attractor, which is a member of the 2D-grid scroll attractor family. However, it should be noted that the system presented here is simpler than the other one. Moreover, the $2 \times 2$-scroll grid attractor is only one member of the scroll grid attractor family. It will be shown that this family can be extended by adding a simple nonlinearity. Another comparable attractor family are $n$-double scroll hypercubes [Suykens \& Chua, 1997] which occur in weak unidirectional or diffusive coupling of $n$-double scroll cells within one-dimensional Cellular Neural Networks [Chua \& Roska, 1993]. However, this family produces hyperchaotic behavior and the order of the system is much higher than the third-order circuit proposed in this paper. From a system and control theo- retical point of view, the proposed system can be represented as a Lur'e system. Hence, many results concerning stability and synchronization are applicable to it [Vidyasagar, 1993; Khalil, 1993; Suykens et al., 1999]. From a circuit design point of view, the new circuit is easily realized by using simple comparators. Moreover, it is possible to systematically increase the complexity of the circuit, by simply using additional core nonlinearities. From an application point of view, this system can produce more complicated signals. Hence it is promising in many applications for chaotic systems as communications and cryptosystems.

This paper is organized as follows. In Sec. 2 we present a generalized chaos generator which produces 1D-grid scroll attractors. 2D- and 3D-grid scroll attractors are presented in Secs. 3 and 4, respectively. In Sec. 5 Lur'e representations for the systems are given. Finally, in Sec. 6 the realization of some of $1 \mathrm{D}$-, 2D- and 3D-grid scroll attractors is given.

\section{A New Family of $n$-Scroll Attractors}

A simple chaos generator model has been recently proposed by Elwakil et al. [2000] which is described by

$$
\dot{\mathbf{x}}=\mathbf{A x}+\mathbf{B} \Phi(\mathbf{x})
$$

with

$$
\begin{aligned}
& \mathbf{A}=\left[\begin{array}{rrr}
0 & 1 & 0 \\
0 & 0 & 1 \\
-a & -a & -a
\end{array}\right], \quad \mathbf{B}=\left[\begin{array}{lll}
0 & 0 & 0 \\
0 & 0 & 0 \\
0 & 0 & a
\end{array}\right], \\
& \Phi=\left[\begin{array}{c}
0 \\
0 \\
f_{1}(x)
\end{array}\right]
\end{aligned}
$$

where

$$
f_{1}(\zeta)=\left\{\begin{aligned}
1, & \zeta \geq 0 \\
-1, & \zeta<0
\end{aligned}\right.
$$

and $\mathbf{x}=[x ; y ; z] \in \mathbb{R}^{3}, \zeta \in \mathbb{R}$. In [Elwakil et al., 2000], it has been reported that the model is extremely simple and produces a double scroll-like attractor for $a=0.8$. A generalization of this original model for generating $n$-scrolls has been recently 


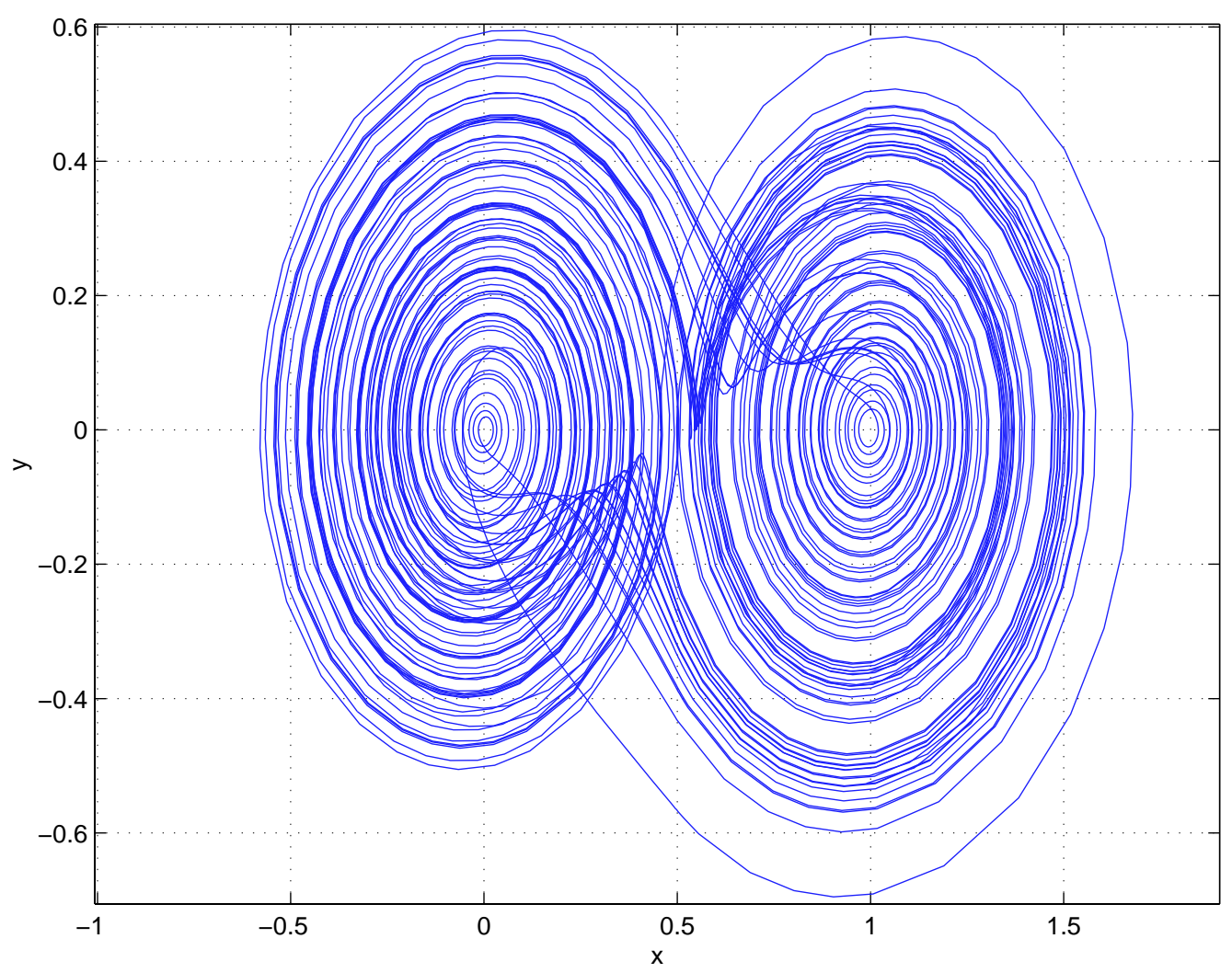

Fig. 1. Double scroll attractor; $f_{1}(x)-g_{0.5}(x), \mathbf{x}_{0}-[0.4565 ; 0.0185 ; 0.8214]$.

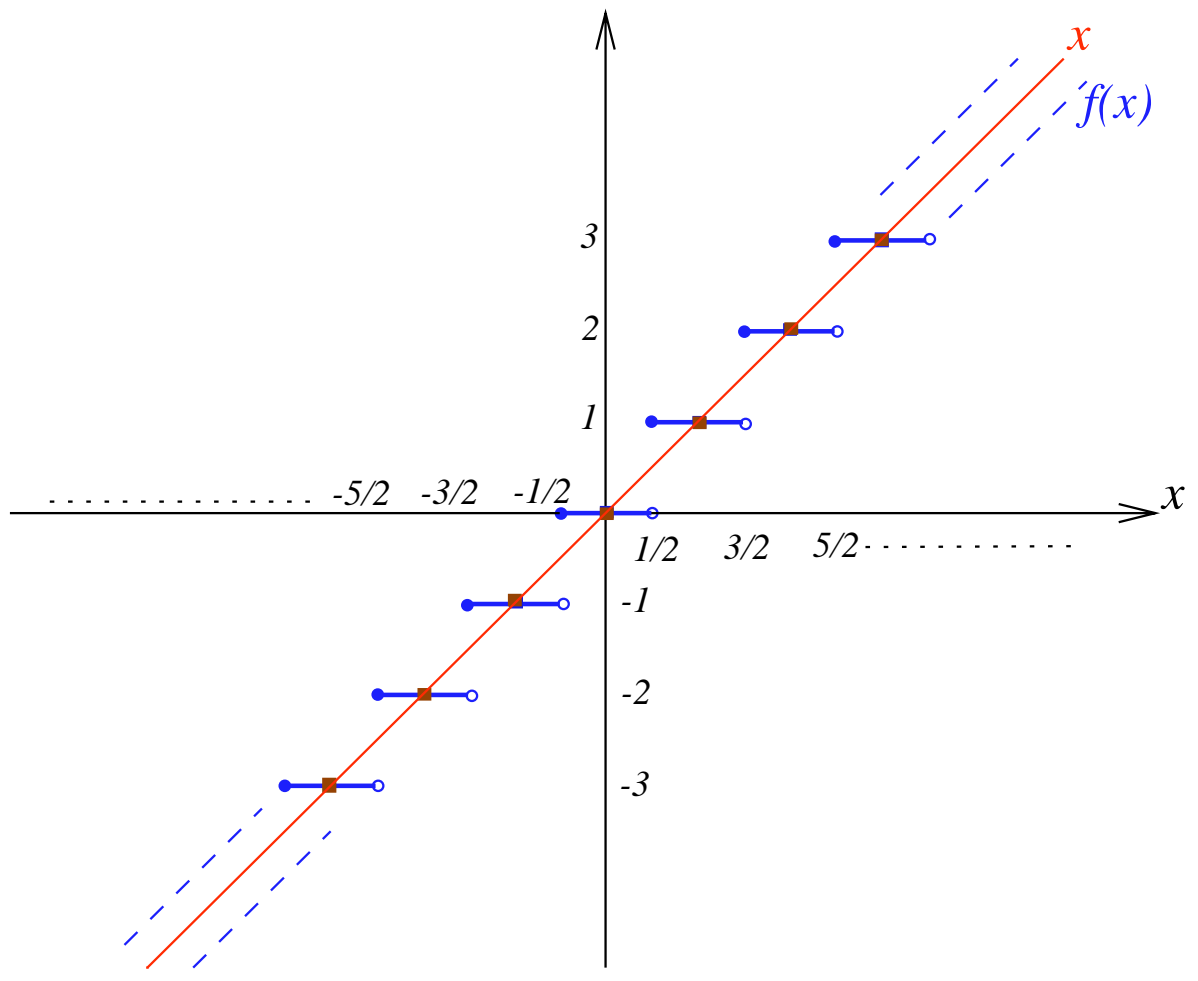

Fig. 2. 1D-grid scroll attractors: the equilibrium points (brown box) are shown at the intersection of $x$ (red solid) and $f(x)$ (blue solid). 
given by Yalçın et al. [2000c]. Here, we consider a minor modification of the latter model by taking the nonlinearity

$$
f_{1}(x)=\sum_{i=1}^{M_{x}} g_{\frac{(-2 i+1)}{2}}(x)+\sum_{i=1}^{N_{x}} g_{\frac{(2 i-1)}{2}}(x)
$$

where

$$
g_{\theta}(\zeta)=\left\{\begin{array}{rcc}
1, & \zeta \geq \theta & \theta>0 \\
0, & \zeta<\theta & \theta>0 \\
0, & \zeta \geq \theta & \theta<0 \\
-1, & \zeta<\theta & \theta<0 .
\end{array}\right.
$$

A computer simulation for the double scroll attractor is shown in Fig. 1 corresponding to $M_{x}=0$,

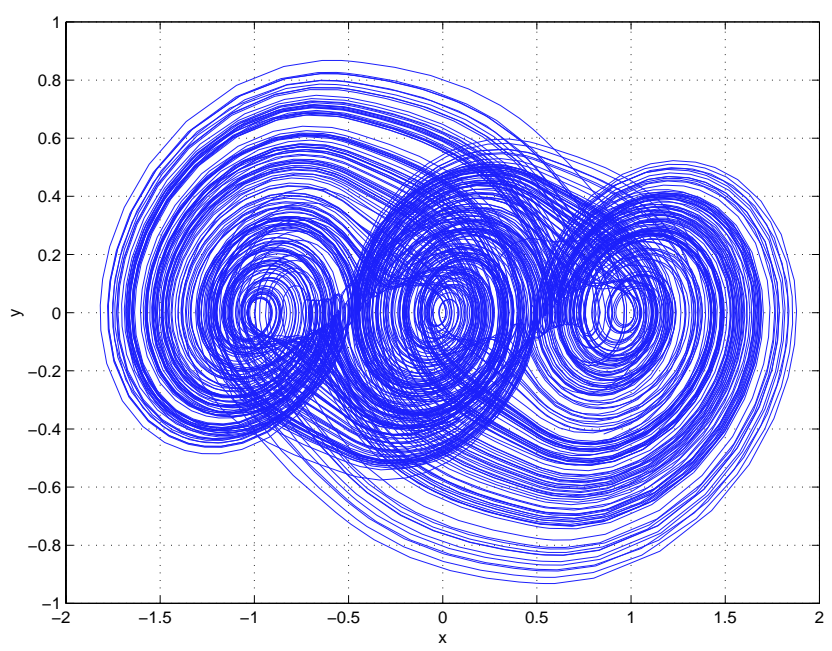

(a)
$N_{x}=1$ and $a=0.8$. A generalization of the system Eq. (1) can be systematically obtained by introducing additional breakpoints in the nonlinearity where each breakpoint can be implemented by Eq. (4). Therefore, we call Eq. (4) the core function. The equilibrium points can be found from the following set of equations

$$
\left\{\begin{array}{l}
x=f_{1}(x) \\
y=0 \\
z=0
\end{array}\right.
$$

The equilibrium points are located at the intersection of the nonlinear function $f_{1}(x)$ and $x$ drawn in

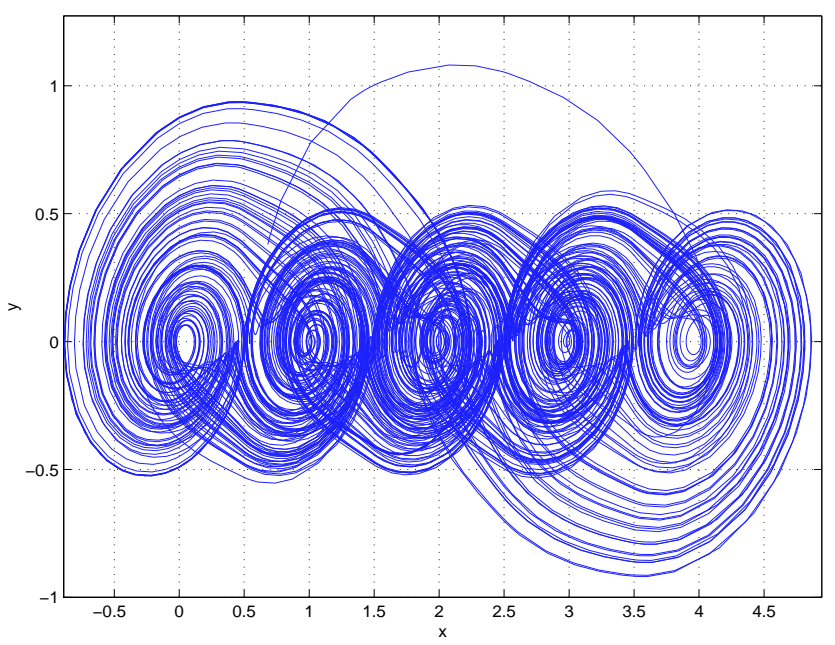

(b)

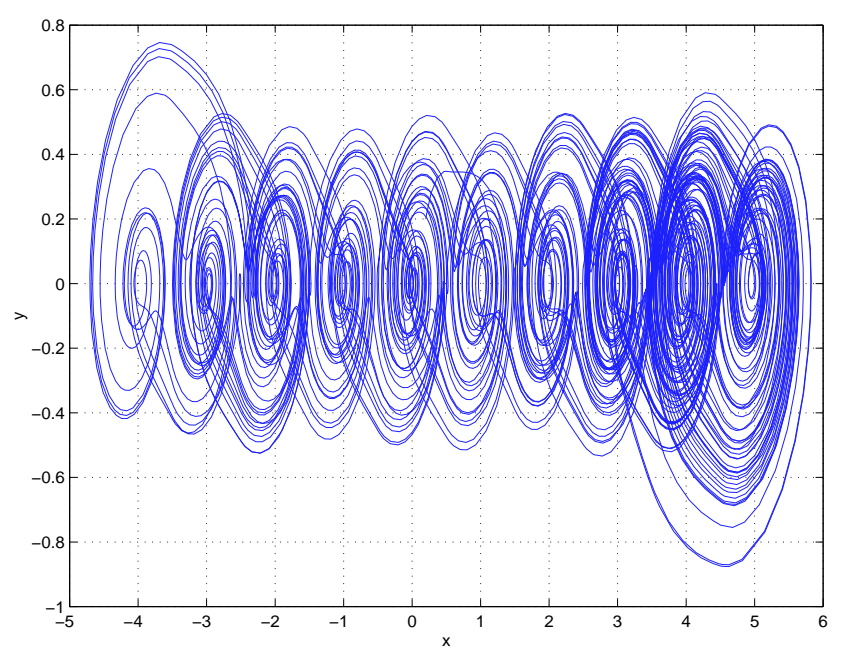

(c)

Fig. 3. (a) $M=1, N=1,3$-scroll attractor, $\mathbf{x}_{0}=[0.30460 ; 18970 ; 1934]$; (b) $M=0, N=4$, 5 -scroll attractor, $\mathbf{x}_{0}=$ [0.3529; 0.8132; 0.0099]; (c) $M=4, N=5,10$-scroll attractor, $\mathbf{x}_{0}=[0.6721 ; 0.8381 ; 0.0196]$. 


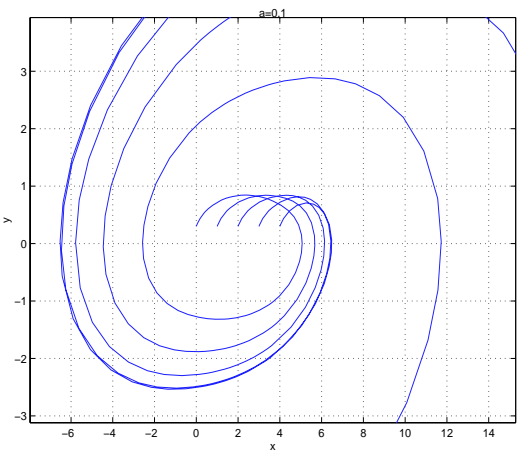

(a)

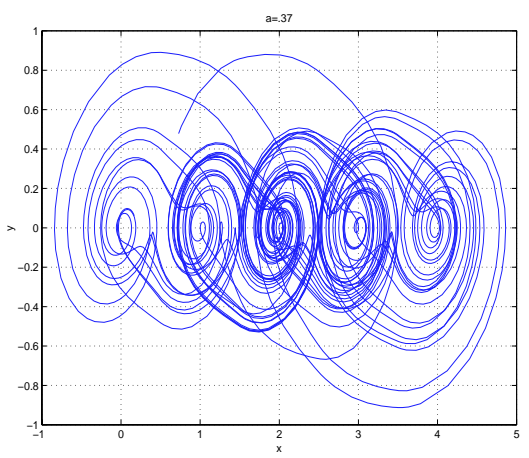

(d)

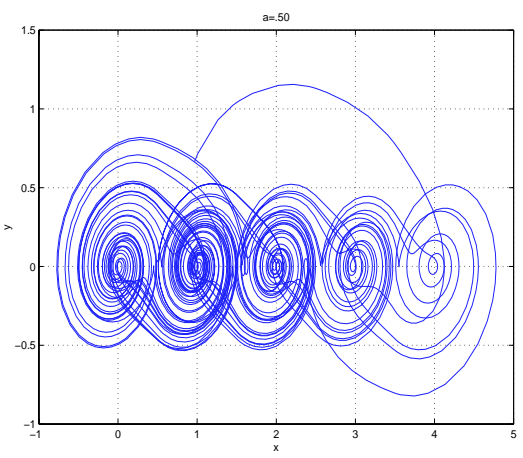

(g)

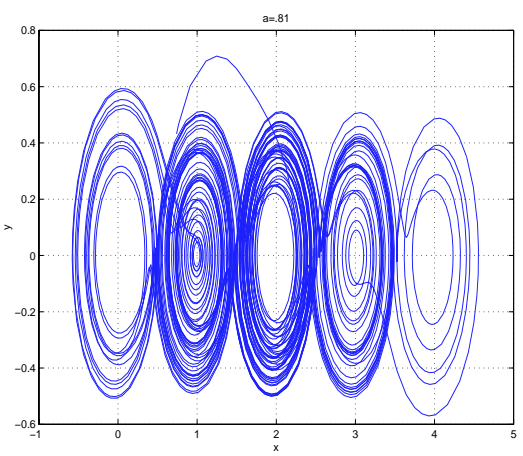

(j)

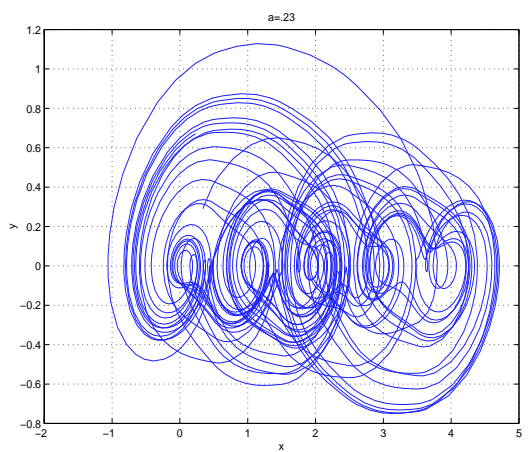

(b)

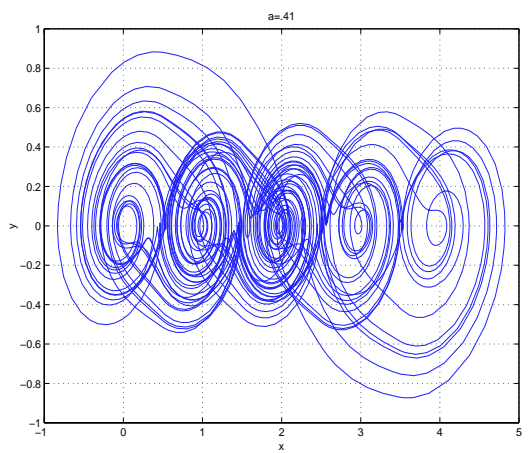

(e)

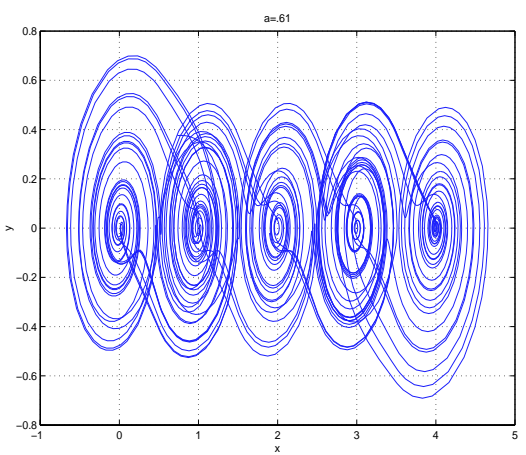

(h)

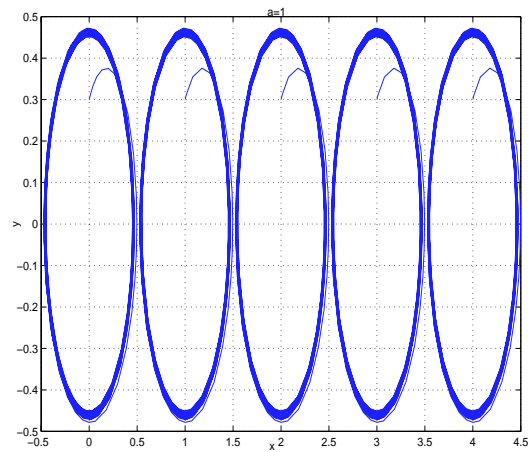

(k)

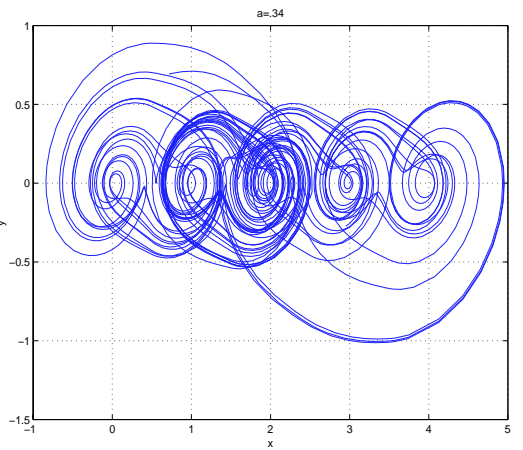

(c)

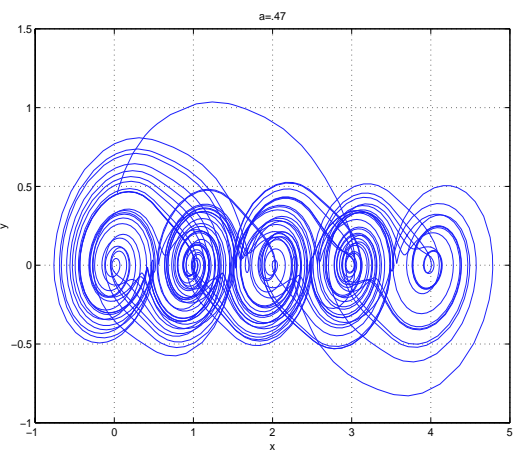

(f)

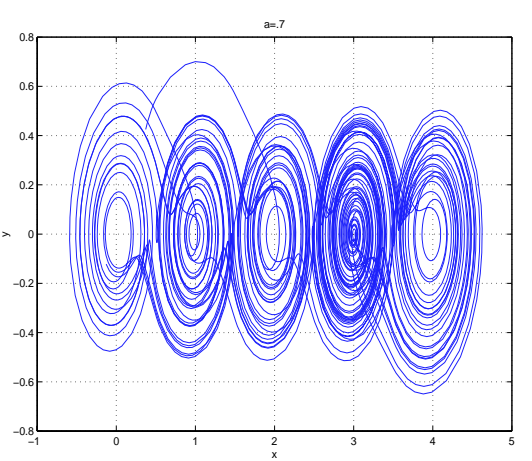

(i)

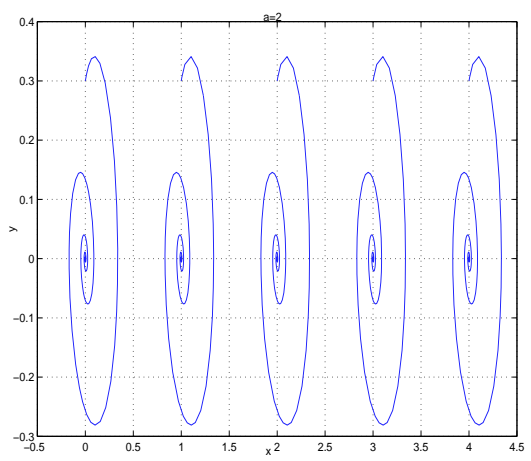

(1)

Fig. 4. Bifurcations related to 5 -scroll attractors with respect to parameter value $a$ : (a) $a=0.1$, for five different initial conditions which are close to the equilibrium points; (b) $a=0.23$; (c) $a=0.34$; (d) $a=0.37$; (e) $a=0.41$; (f) $a=0.47$; (g) $a=0.5$; (h) $a=0.61$; (i) $a=0.7$; (j) $a=0.81$, for five different initial conditions which are close to the equilibrium points; (k) $a=1 ;(1) a=2$. 
Fig. 2 for the general case. The set of equilibrium points is

$\mathcal{V}_{\text {eq }}=\left\{[i ; 0 ; 0] \mid i=-M_{x},-M_{x}+1, \ldots, N_{x}-1, N_{x}\right\}$

which are located on the $x$-axis in state space [Fig. 8(a)]. Due to the location of the equilibrium points, this strange attractor family is called 1Dgrid scroll attractors. In the attractors, the scrolls are located around the equilibrium points. Therefore, the number of scrolls equals the number of equilibrium points. The number of scrolls generated from the generalized nonlinearity is equal to $M_{x}+N_{x}+1$. In Fig. 3, 3-, 5- and 10-scroll attractors are shown by using the generic model for $a=0.4$ and for $\left(M_{x}=1, N_{x}=1\right),\left(M_{x}=0\right.$, $\left.N_{x}=4\right),\left(M_{x}=4, N_{x}=5\right)$, respectively. Figure 4 shows the bifurcation phenomenon with respect to the parameter value $a$ related to a 5 -scroll attractor.

\section{2D-Grid Scroll Attractors}

Now we show that it is also possible to increase the number of scrolls in the $y$ state variable direction. We start from the same system (1), but with

$$
\begin{aligned}
\mathbf{A} & =\left[\begin{array}{rrr}
0 & 1 & 0 \\
0 & 0 & 1 \\
-a & -a & -a
\end{array}\right], \quad \mathbf{B}=\left[\begin{array}{rrr}
-1 & 0 & 0 \\
0 & 0 & 0 \\
0 & 0 & a
\end{array}\right], \\
\Phi & =\left[\begin{array}{c}
f_{1}(y) \\
0 \\
f_{2}(x)
\end{array}\right]
\end{aligned}
$$

with

$$
f_{1}(y)=\sum_{i=1}^{M_{y}} g_{\frac{(-2 i+1)}{2}}(y)+\sum_{i=1}^{N_{y}} g_{\frac{(2 i-1)}{2}}(y)
$$

and additional nonlinearity

$$
f_{2}(x)=\sum_{i=1}^{m-1} \beta g_{p_{i}}(x)
$$

where

$$
\begin{aligned}
p_{i} & =M_{y}+0.5+(i-1)\left(M_{y}+N_{y}+1\right) \\
\beta & =M_{y}+N_{y}+1 .
\end{aligned}
$$

The equilibrium points follow from

$$
\left\{\begin{array}{l}
x+y=f_{2}(x) \\
y=f_{1}(y) \\
z=0
\end{array}\right.
$$

The solutions of the second equation of (7) have already been indicated in the previous section, which was given by

$$
u^{\mathrm{eq}, y}=\left[-M_{y} ; \ldots ;-1 ; 0 ; 1 ; \ldots N_{y}\right] .
$$

The points for the $x$ state variable corresponding to each $u_{j}^{\mathrm{e} q, y}$ are determined in a graphyical way from the first equation of (7). The set of equilibrium points becomes

$$
\begin{aligned}
\mathcal{V}_{\mathrm{eq}} & =\left\{\left[(i-1)\left(M_{y}+N_{y}+1\right)+j,-j ; 0\right]\right. \\
\quad \mid i & \left.=1,2, \ldots, m ; j=-N_{y}, \ldots,-1,0,1, \ldots, M_{y}\right\}
\end{aligned}
$$

It should be noted that the locations of the equilibrium points are located in the $x-y$ plane [Fig. 8(b)] and the system has a number of $m\left(M_{y}+N_{y}+1\right)$ equilibrium points. For this reason, we will call this strange attractor family $m \times\left(M_{y}+N_{y}+1\right)$-scroll grid attractors. Some resulting 2D-grid scroll attractors and their corresponding nonlinearities are given by:

- $2 \times 2$-grid scroll attractor $\left(M_{y}=0, N_{y}=1\right.$, $m=2$ ) (Fig. 5)

$$
\begin{aligned}
& f_{1}(y)=g_{0.5}(y) \\
& f_{2}(x)=2 g_{0.5}(x)
\end{aligned}
$$

- $2 \times 3$-grid scroll attractor $\left(M_{y}=0, N_{y}=2\right.$, $m=2$ ) (Fig. 6)

$$
\begin{aligned}
& f_{1}(y)=g_{0.5}(y)+g_{1.5}(y) \\
& f_{2}(x)=3 g_{0.5}(x)
\end{aligned}
$$

- $3 \times 3$-grid scroll attractor $\left(M_{y}=0, N_{y}=2\right.$, $m=3)$ [Fig. 7(a)]

$$
\begin{aligned}
& f_{1}(y)=g_{0.5}(y)+g_{1.5}(y) \\
& f_{2}(x)=3\left(g_{0.5}(x)+g_{3.5}(x)\right)
\end{aligned}
$$

- $4 \times 4$-grid scroll attractor $\left(M_{y}=0, N_{y}=3\right.$, $m=4)$ [Fig. 7(b)]

$$
\begin{aligned}
& f_{1}(y)=g_{0.5}(y)+g_{1.5}(y)+g_{2.5}(y) \\
& f_{2}(x)=4\left(g_{0.5}(x)+g_{4.5}(x)+g_{8.5}(x)\right)
\end{aligned}
$$

- $4 \times 5$-grid scroll attractor $\left(M_{y}=0, N_{y}=4\right.$, $m=4)$ [Fig. 7(c)]

$$
\begin{aligned}
& f_{1}(y)=g_{0.5}(y)+g_{1.5}(y)+g_{2.5}(y)+g_{3.5}(y) \\
& f_{2}(x)=5\left(g_{0.5}(x)+g_{5.5}(x)+g_{10.5}(x)\right)
\end{aligned}
$$




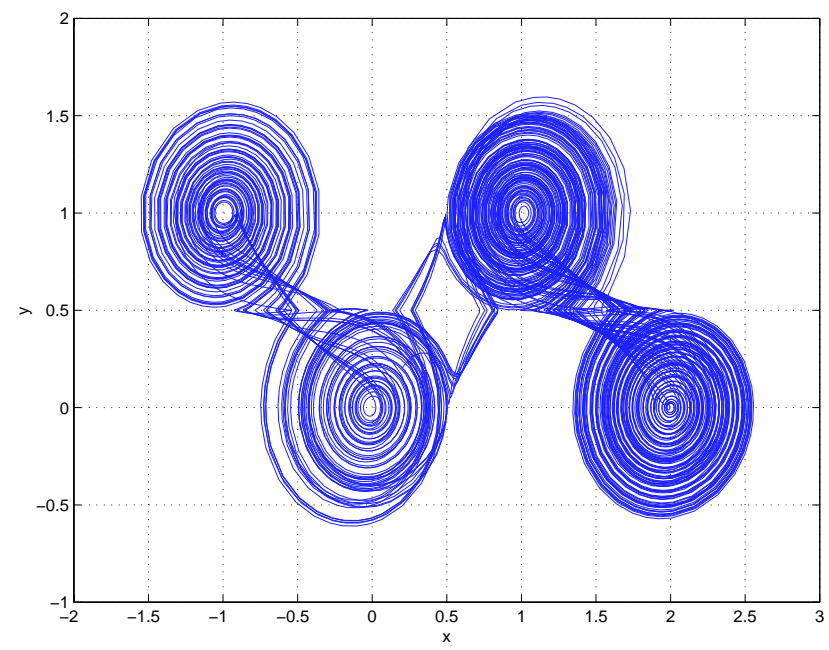

(a)

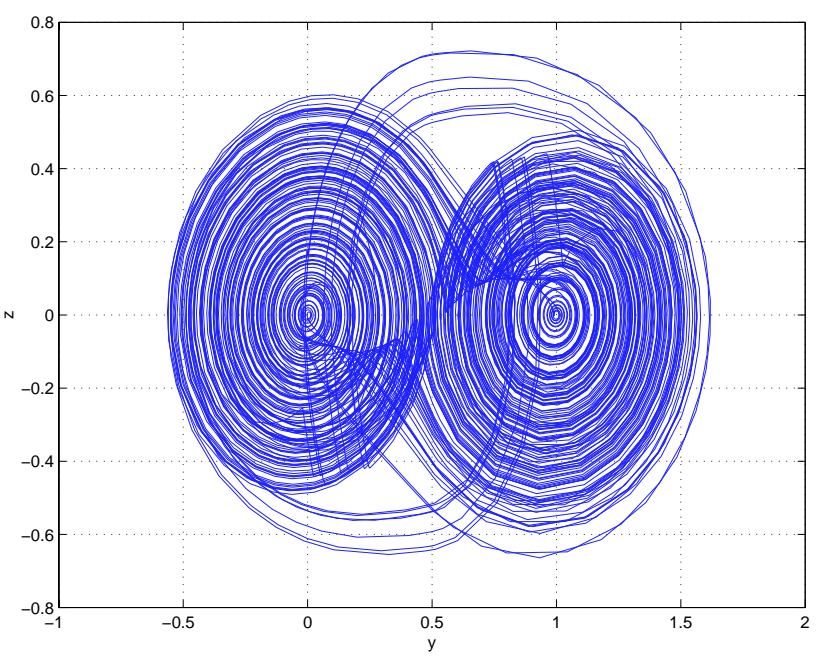

(b)

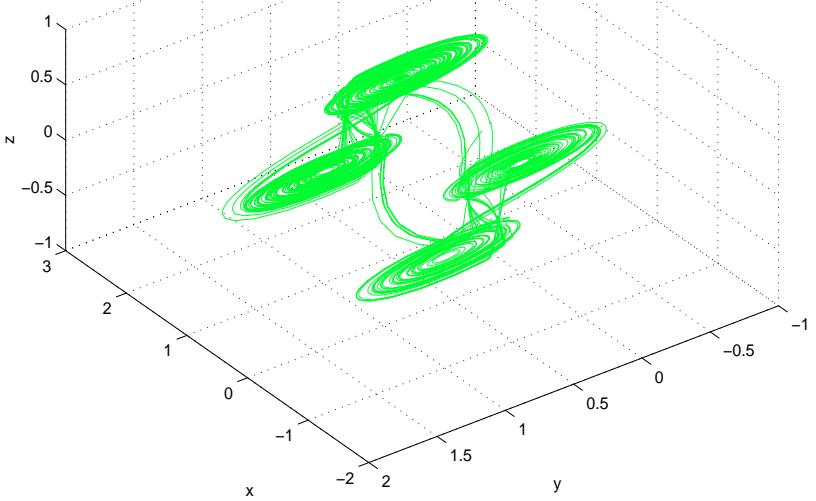

(c)

Fig. 5. $2 \times 2$-grid scroll attractor. Projection onto (a) the $(x, y)$ and $(\mathrm{b})$ the $(y, z)$ planes, (c) view on 3D-state space.

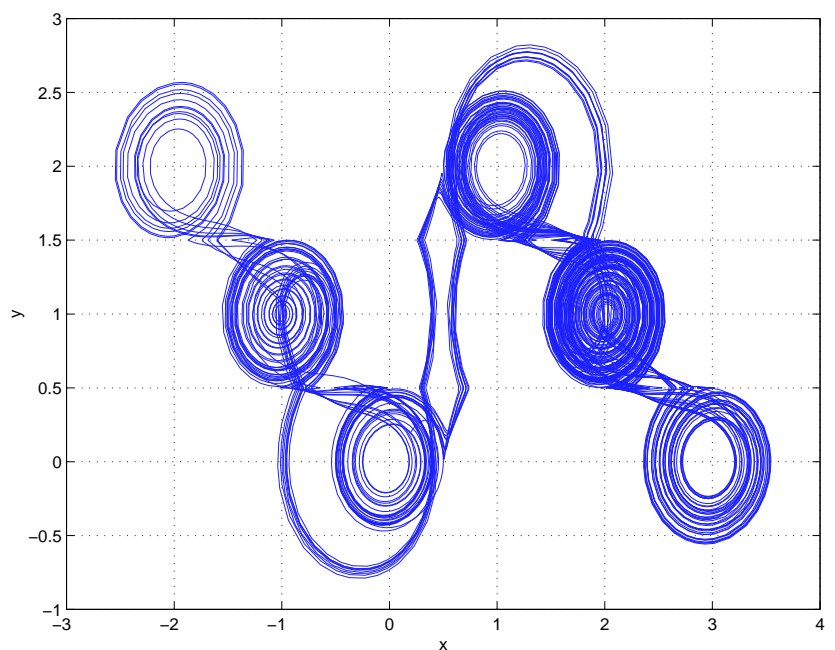

(a)

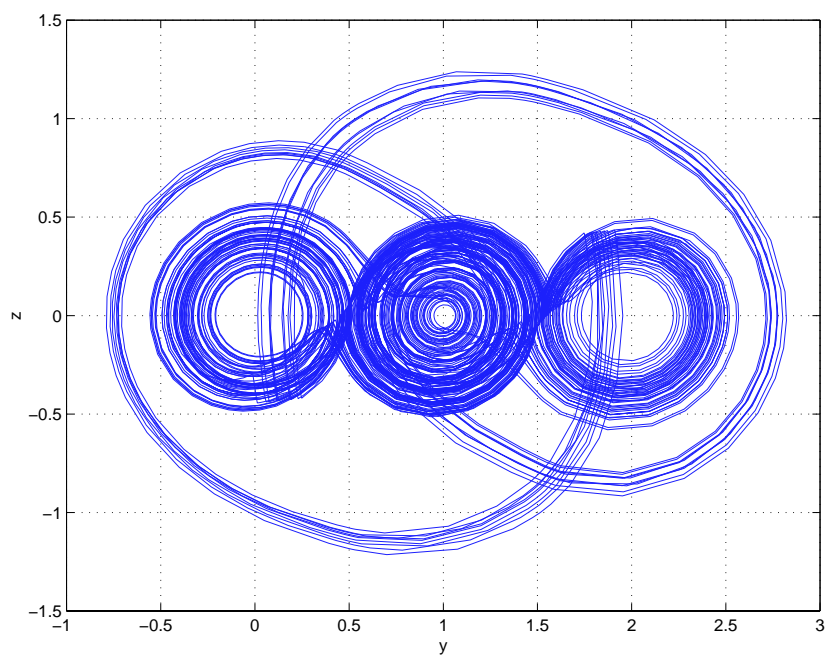

(b)

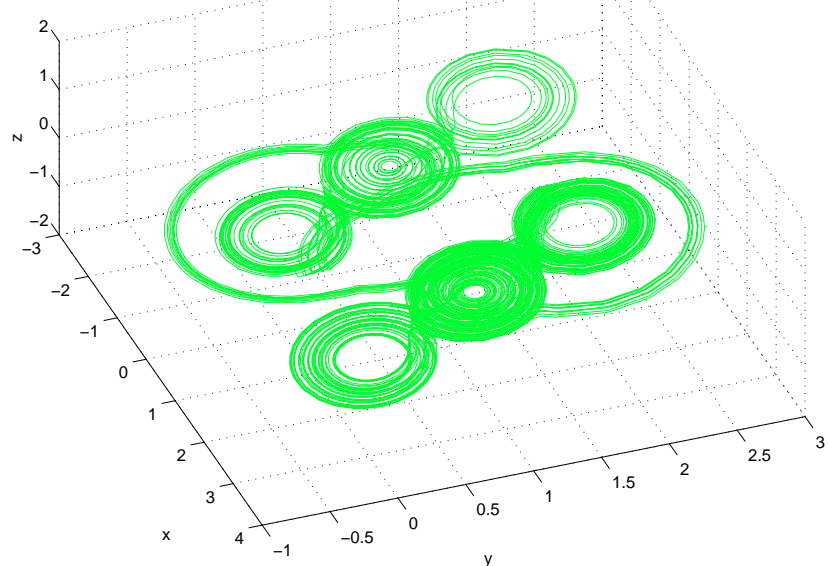

(c)

Fig. 6. $2 \times 3$-grid scroll attractor. 


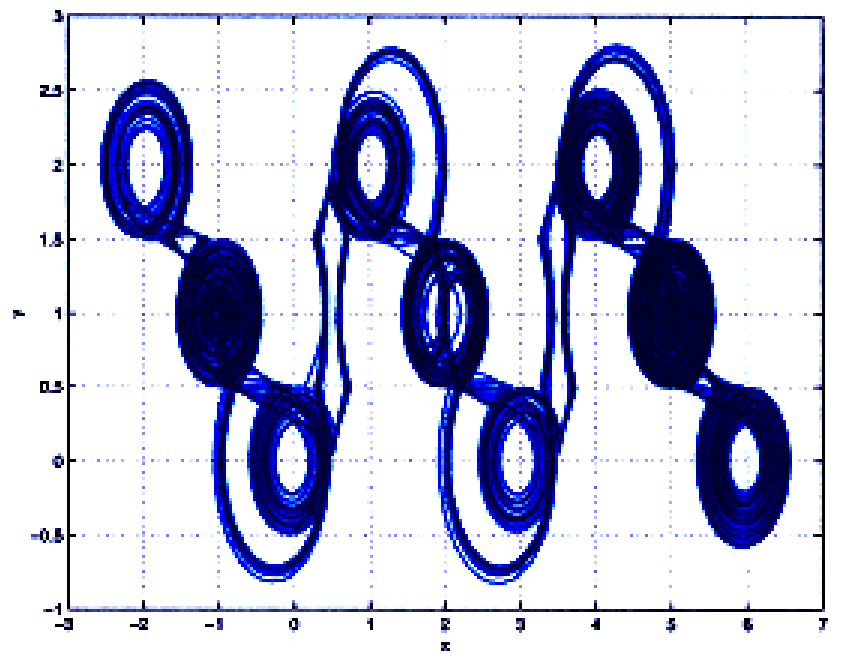

(a)

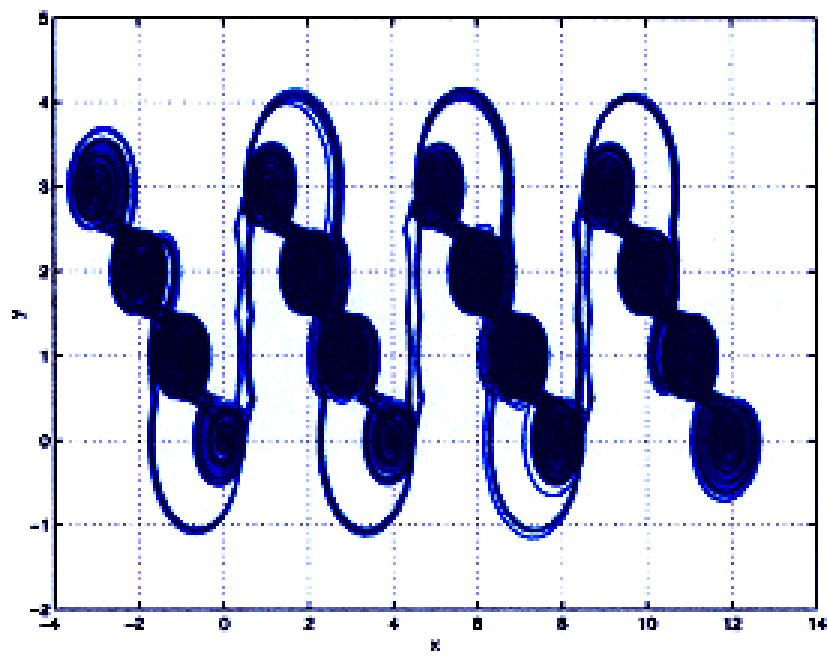

(b)

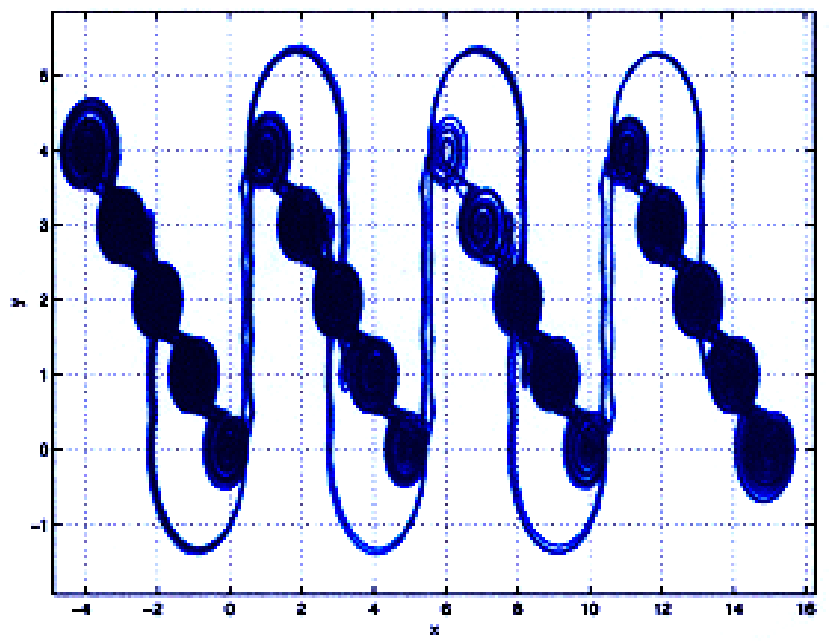

(c)

Fig. 7. Projection onto the $(x, y)$ plane of (a) $3 \times 3-$, (b) $4 \times 4$ and (c) $4 \times 5$-grid scroll attractor.

\section{3D-Grid Scroll Attractors}

The following additional nonlinearity $f_{1}(z)$ is introduced now to the system (1) with

$$
\begin{aligned}
& \mathbf{A}=\left[\begin{array}{rrr}
0 & 1 & 0 \\
0 & 0 & 1 \\
-a & -a & -a
\end{array}\right], \quad \mathbf{B}=\left[\begin{array}{rrr}
-1 & 0 & 0 \\
0 & -1 & 0 \\
0 & 0 & a
\end{array}\right], \\
& \Phi=\left[\begin{array}{l}
f_{1}(y) \\
f_{1}(z) \\
f_{3}(x)
\end{array}\right]
\end{aligned}
$$

where

$$
f_{1}(z)=\sum_{i=1}^{M_{z}} g_{\frac{(-2 i+1)}{2}}(z)+\sum_{i=1}^{N_{z}} g_{\frac{(2 i-1)}{2}}(z),
$$

and

$$
f_{3}(x)=\sum_{l=1}^{k-1} \gamma g_{n_{l}}(x)
$$

where

$$
\begin{aligned}
n_{l} & =\rho+0.5+(l-1)(\rho+\varsigma+1) \\
\gamma & =\rho+\varsigma+1
\end{aligned}
$$

with

$$
\begin{aligned}
\rho & =\left|\min _{i, j}\left\{u_{i}^{\mathrm{eq}, y}+u_{j}^{\mathrm{eq}, z}\right\}\right|, \\
\varsigma & =\left|\max _{i, j}\left\{u_{i}^{\mathrm{eq}, y}+u_{j}^{\mathrm{eq}, z}\right\}\right|
\end{aligned}
$$

and $u^{\mathrm{eq}, y}$ and $u^{\mathrm{eq}, z}$ are the vectors for the $y$ and $z$ variables related to the equilibrium points. The equilibrium points follow from

$$
\left\{\begin{array}{l}
x+y+z=f_{3}(x) \\
y=f_{1}(y) \\
z=f_{1}(z)
\end{array}\right.
$$

where the points for the $y, z$ variables are given by

$$
\begin{aligned}
& u^{\mathrm{eq}, y}=\left[-M_{y} ; \ldots ;-1 ; 0 ; 1 ; \ldots ; N_{y}\right], \\
& u^{\mathrm{eq}, z}=\left[-M_{z} ; \ldots ;-1 ; 0 ; 1 ; \ldots ; N_{z}\right] .
\end{aligned}
$$

With these nonlinearities the system produces $k \times$ $\left(M_{y}+N_{y}+1\right) \times\left(M_{z}+N_{z}+1\right)$-scroll grid attractors. 
All the scrolls are located around the equilibrium points which are given

$$
\begin{aligned}
\mathcal{V}_{\mathrm{eq}}=\{[ & (l-1)(\varsigma+1+\rho)-u_{i}^{\mathrm{eq}, y} \\
& \left.\left.-u_{j}^{\mathrm{eq}, z} ; u_{i}^{\mathrm{eq}, y} ; u_{j}^{\mathrm{eq}, z}\right]\right\}
\end{aligned}
$$

with $i=1,2, \ldots, M_{y}+N_{y}+1, j=1,2, \ldots, M_{z}+$ $N_{z}+1$ and $l=1,2, \ldots, k$. The location of the equilibrium points in $3 \mathrm{D}$ is shown in Fig. 8(c). Here, some examples of 3D-grid scroll attractors are given:

- $2 \times 2 \times 2$-grid scroll attractor $\left(M_{y}=0, N_{y}=1\right.$, $M_{z}=0, N_{z}=1, k=2$ ) (Fig. 9)

$$
\begin{aligned}
& f_{3}(x)=3 g_{0.5}(x) \\
& f_{1}(y)=g_{0.5}(y) \\
& f_{1}(z)=g_{0.5}(z)
\end{aligned}
$$

- $4 \times 2 \times 2$-grid scroll attractor $\left(M_{y}=0, N_{y}=1\right.$, $\left.M_{z}=0, N_{z}=1, k=4\right)$ (Fig. 10)

$$
\begin{aligned}
& f_{3}(x)=3\left(g_{0.5}(x)+g_{3.5}(x)+g_{6.5}(x)\right) \\
& f_{1}(y)=g_{0.5}(y) \\
& f_{1}(z)=g_{0.5}(z)
\end{aligned}
$$

- $4 \times 3 \times 2$-grid scroll attractor $\left(M_{y}=1, N_{y}=1\right.$, $\left.M_{z}=0, N_{z}=1, k=4\right)$ (Fig. 11)

$$
\begin{aligned}
& f_{3}(x)=4\left(g_{1.5}(x)+g_{5.5}(x)+g_{9.5}(x)\right) \\
& f_{1}(y)=g_{-0.5}(y)+g_{0.5}(y) \\
& f_{1}(z)=g_{0.5}(z)
\end{aligned}
$$

\section{Lur'e Representation}

The new circuits from which the scroll grid attractor families are generated can be represented as Lur'e systems, i.e. as a linear system interconnected by feedback to a static nonlinearity that satisfies a sector condition

$$
\dot{\mathbf{x}}=\mathbf{A x}+\mathbf{B} \sigma(\mathbf{C x})
$$

with

$$
\begin{aligned}
& \mathbf{A}=\left[\begin{array}{rrr}
0 & 1 & 0 \\
0 & 0 & 1 \\
-a & -a & -a
\end{array}\right], \quad \mathbf{B}=\left[\begin{array}{ccc}
b_{y} & 0 & 0 \\
0 & b_{z} & 0 \\
0 & 0 & a
\end{array}\right], \\
& \mathbf{C}=\left[\begin{array}{lll}
0 & 1 & 0 \\
0 & 0 & 1 \\
1 & 0 & 0
\end{array}\right]
\end{aligned}
$$

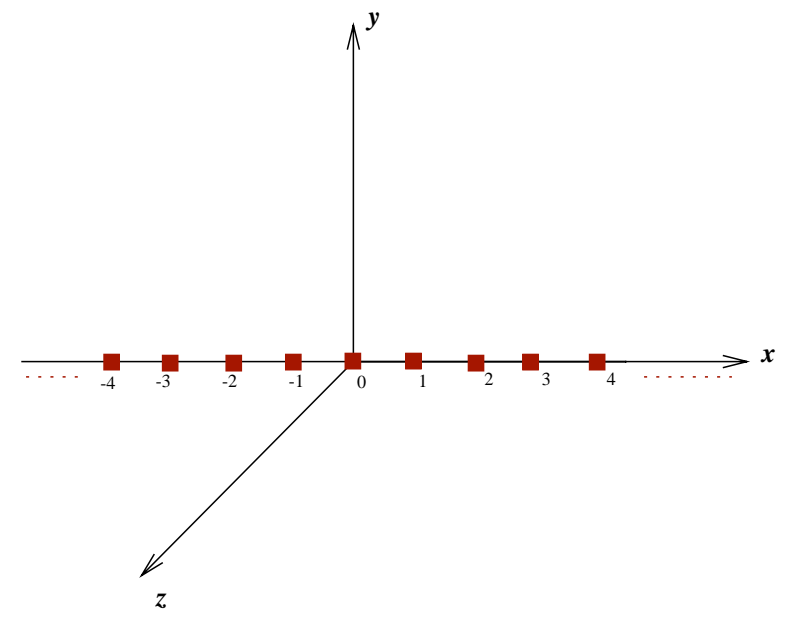

(a)

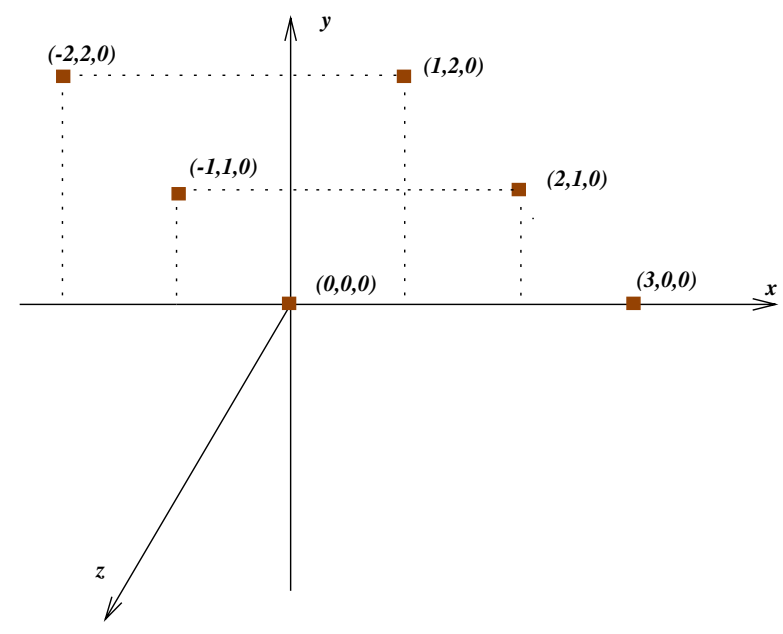

(b)

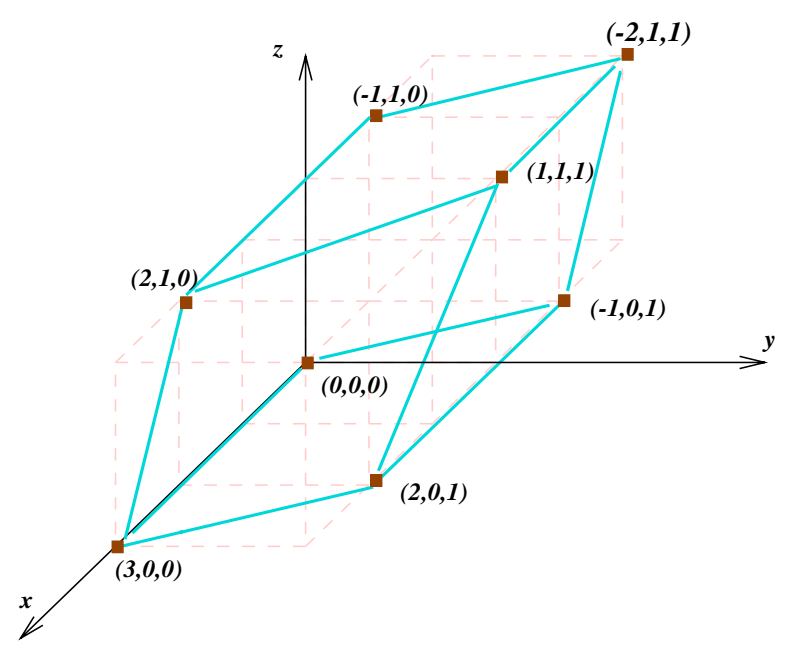

(c)

Fig. 8. The equilibrium points are located on (a) $x$ axis for $1 \mathrm{D}$-grid scroll attractor, (b) $(x, y)$ plane for 2D-grid scroll attractor (equilibrium points are shown for $3 \times 3$-grid attractor) (c) in a $3 \mathrm{D}$ body for $3 \mathrm{D}$-grid scroll attractors (equilibrium points are shown for a $2 \times 2 \times 2$-scroll grid attractor). 


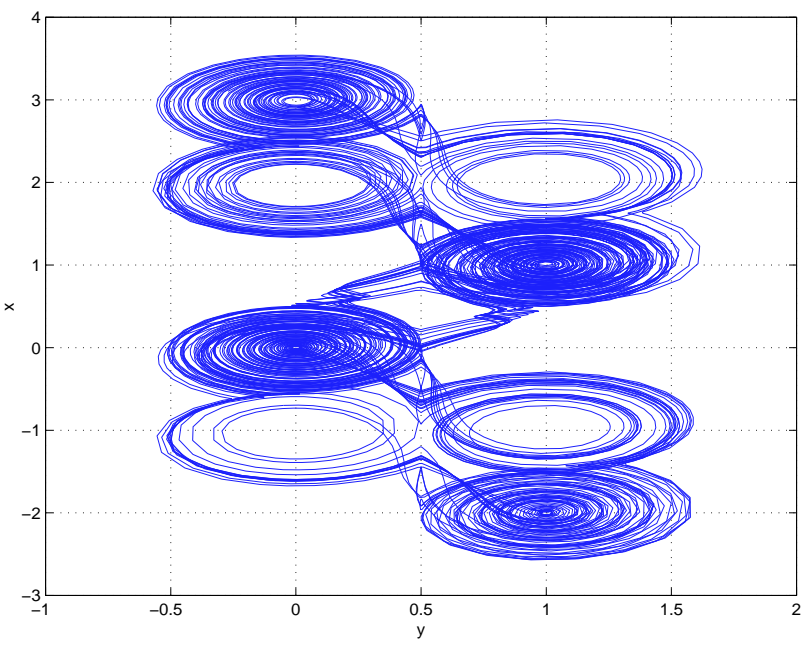

(a)

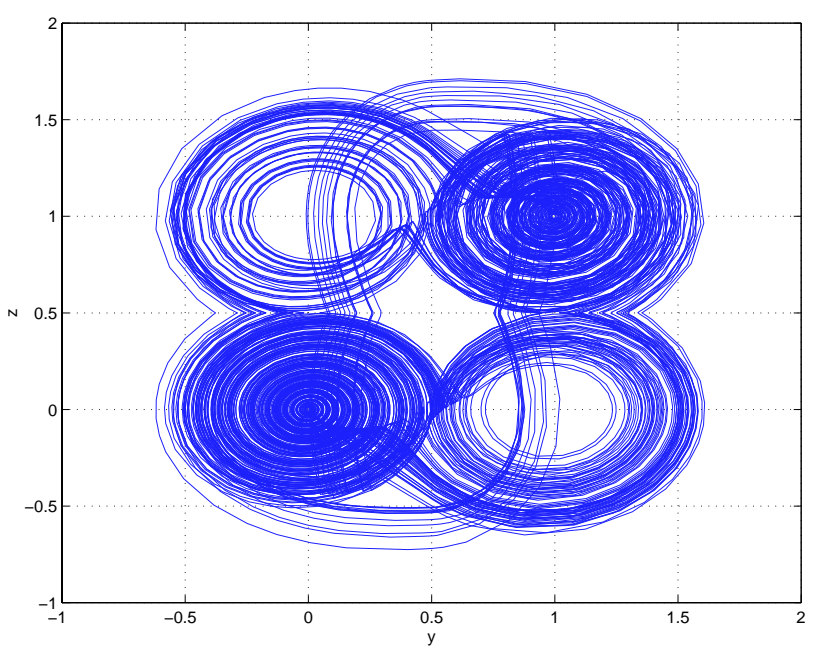

(b)

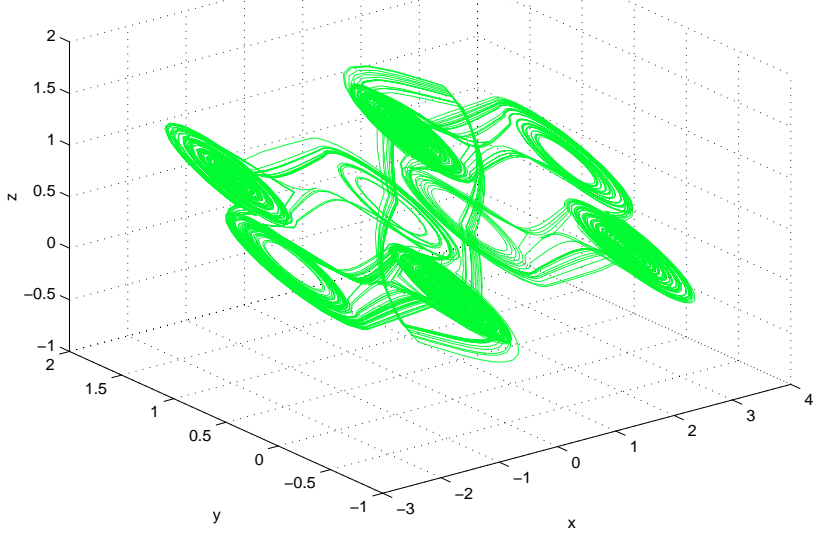

(c)

Fig. 9. $2 \times 2 \times 2$-grid scroll attractor. Projection onto (a) the $(y, x)$ and (b) the $(y, z)$ planes, (c) view on 3D state space.

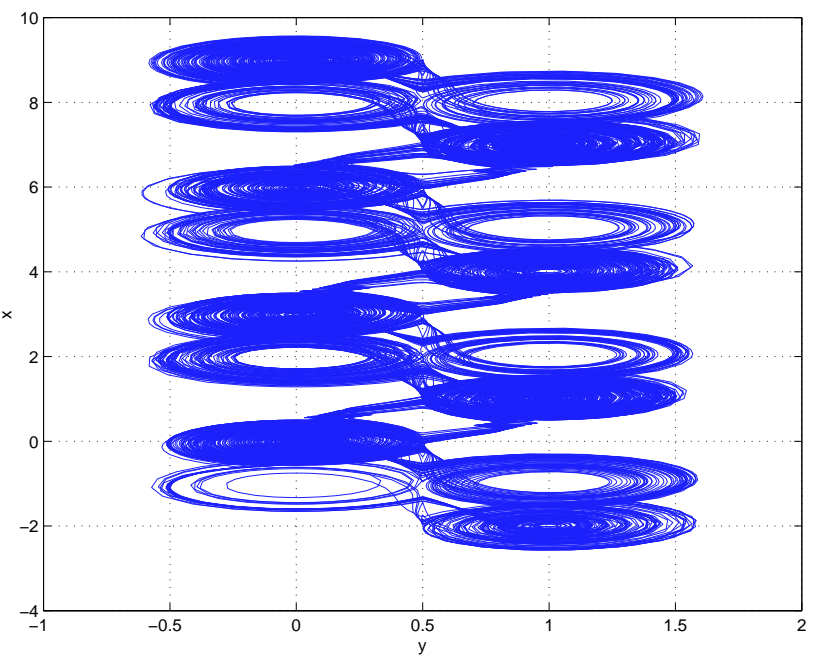

(a)

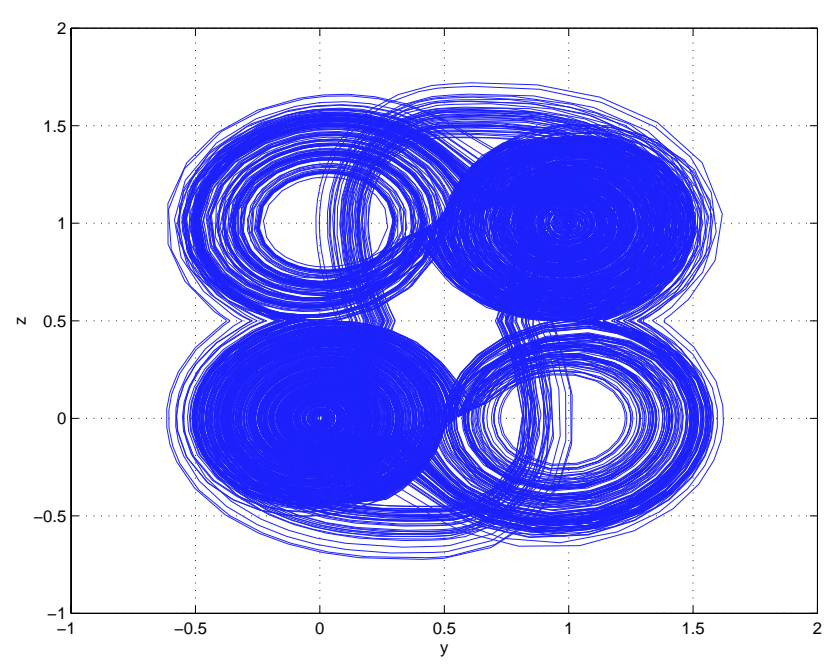

(b)

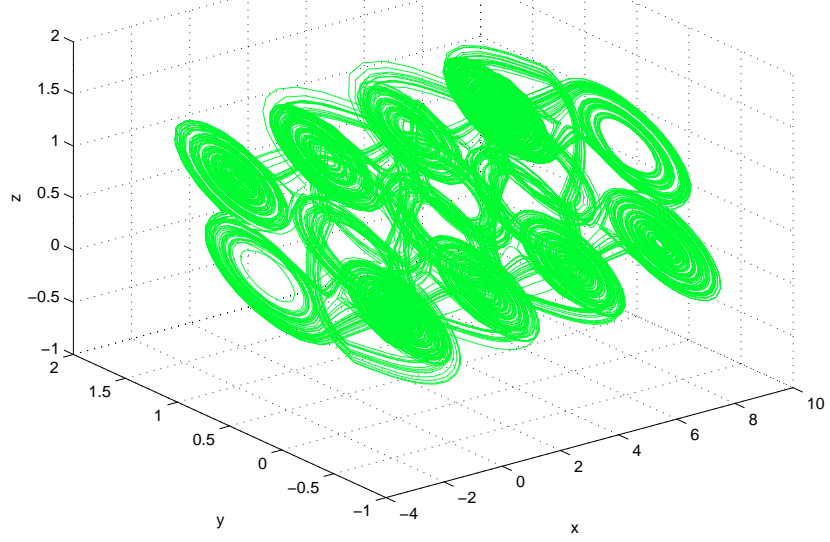

(c)

Fig. 10. $4 \times 2 \times 2$-grid scroll attractor. 


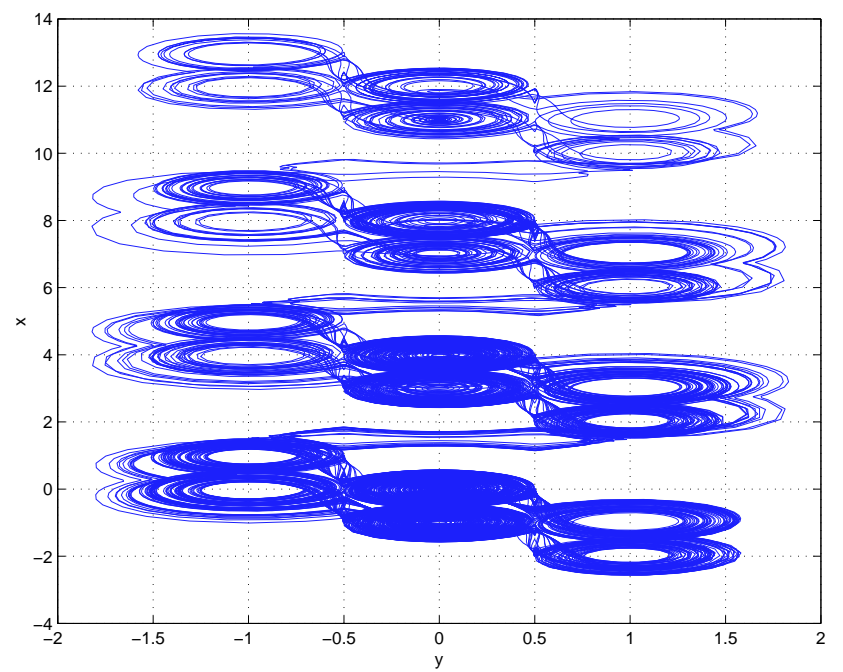

(a)

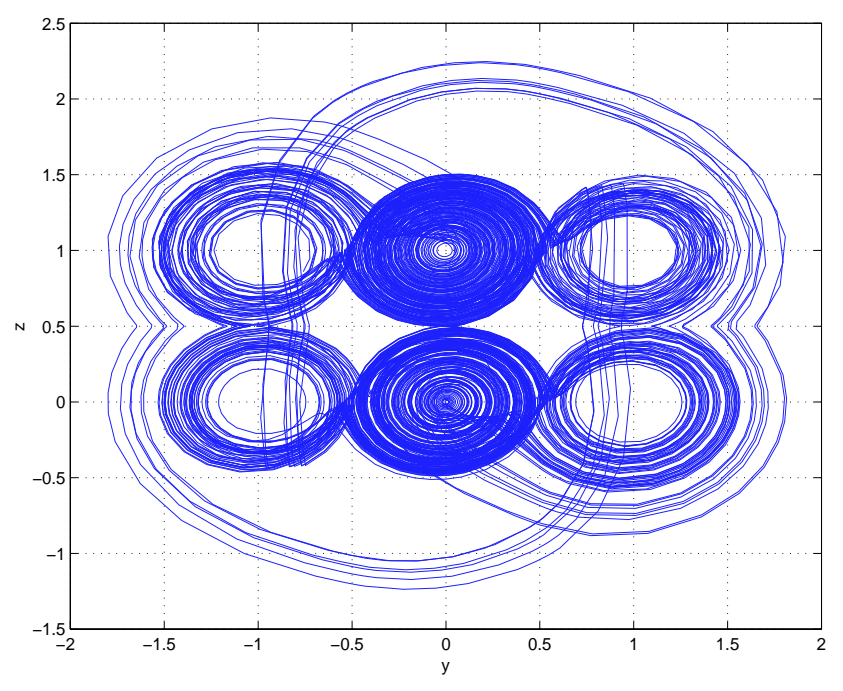

(b)

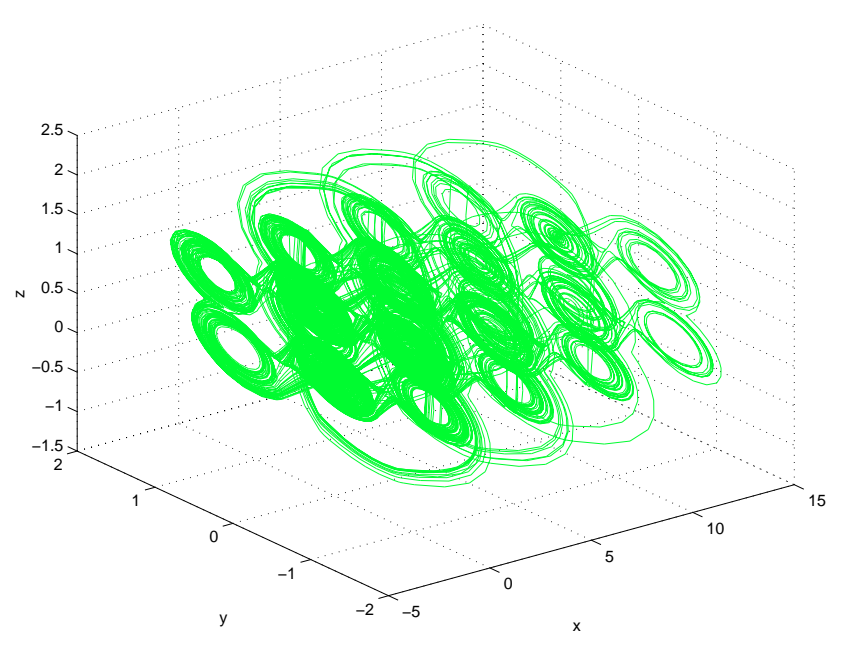

(c)

Fig. 11. $4 \times 3 \times 2$-grid scroll attractor.
One obtains the following for the different cases:

- 1D-scroll grid attractors ( $n$-scroll attractor):

$$
\left\{\begin{array}{c}
b_{y}=0, b_{z}=0 \\
\sigma(\cdot)=\left[\begin{array}{c}
0 \\
0 \\
f_{1}(\cdot)
\end{array}\right]
\end{array}\right.
$$

where $f_{1}(\cdot)$ is given by Eq. (3), which belongs to sector $[0,2]$.

- 2D-scroll grid attractors:

$$
\left\{\begin{array}{l}
b_{y}=-1, b_{z}=0 \\
\sigma(\cdot)=\left[\begin{array}{c}
f_{1}(\cdot) \\
0 \\
f_{2}(\cdot)
\end{array}\right]
\end{array}\right.
$$

where $f_{2}(\cdot)$ is given by Eq. (6), which belongs to sector $\left[0,\left(M_{y}+N_{y}+1\right) /\left(M_{y}+0.5\right)\right]$.

- 3D-grid scroll attractors:

$$
\left\{\begin{array}{c}
b_{y}=-1, b_{z}=-1 \\
\sigma(\cdot)=\left[\begin{array}{l}
f_{1}(\cdot) \\
f_{1}(\cdot) \\
f_{3}(\cdot)
\end{array}\right]
\end{array}\right.
$$

where $f_{3}(\cdot)$ is given by Eq. (9), which belongs to sector $[0,(\varsigma+\rho+1) /(\rho+0.5)]$.

\section{Circuit Realizations}

In this section, the realizations of some of the 1D-, 2D- and 3D-grid scroll attractors discussed above are given. For this purpose, a circuit using conventional voltage opamps could be employed. However, a number of research results, which illustrate the advantages of current feedback opamps (CFOA) over conventional voltage opamps, have been presented in the literature, e.g. [Toumazou et al., 1990; Fabre, 1993; Toumazou \& Lidgey, 1994]. From these works, it is known that CFOA is almost free from slew-rate limitations as opposed to voltage opamp. It is capable of operating at much higher frequencies and offers design flexibility which allows the derivation of relatively simpler circuits. Considering these facts, researchers have attempted to use CFOAs in the implementation of chaotic oscillators in order to have an improved high-frequency performance, e.g. [Senani \& Gupta, 1998; Elwakil 


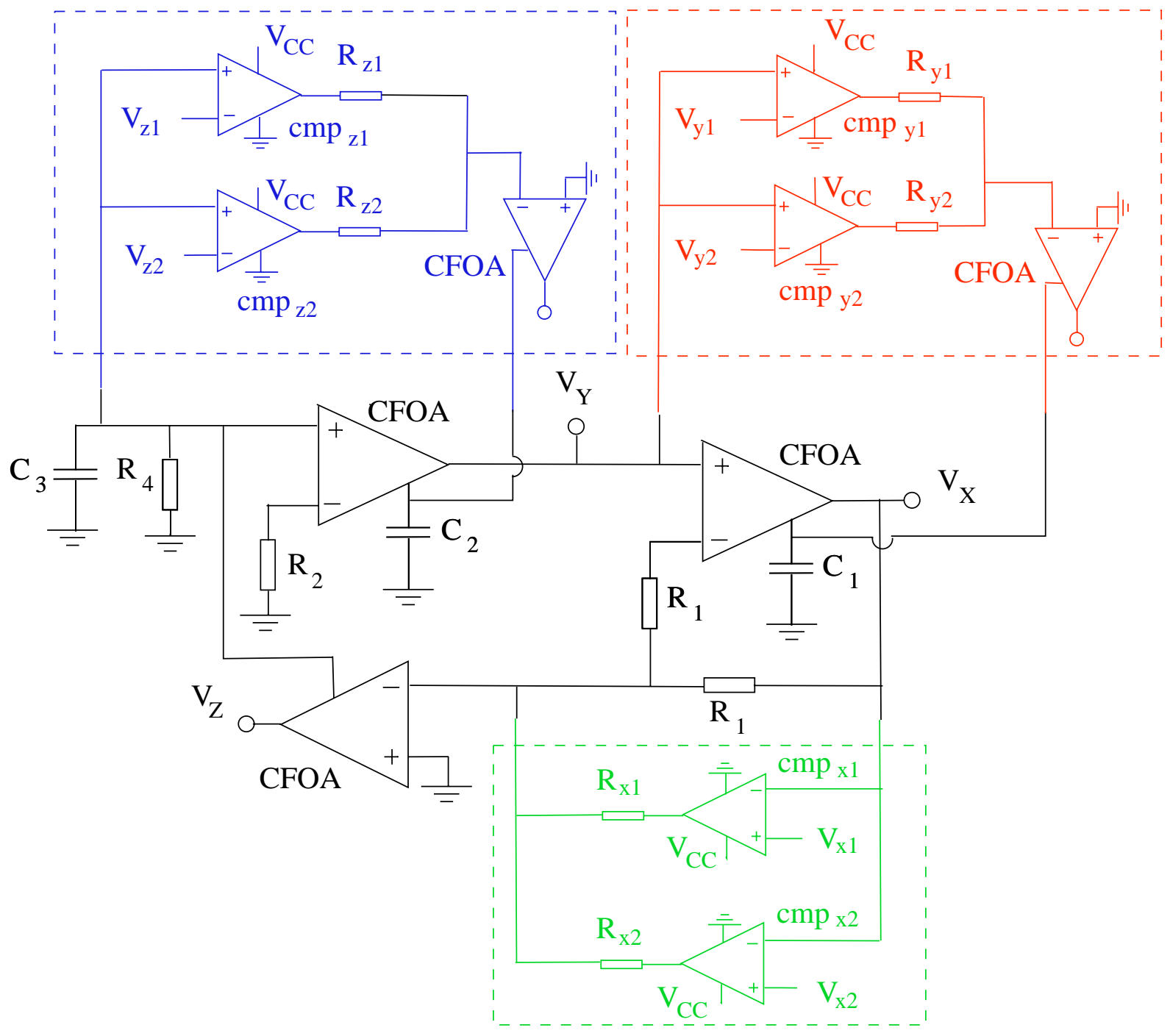

Fig. 12. Possible circuit diagram realizing the proposed system.

\& Kennedy, 1998, 2000]. In the same way, we have also used a possible CFOA-based circuit in order to realize and observe some of the scroll grid attractors described in the previous sections. The circuit we have used for this purpose is given in Fig. 12. The CFOAs implemented using AD $844^{1}$ from Analog Devices and the required nonlinearities in Eqs. (5), (8) and (9) are realized using the subcircuits drawn within the dashed lines. The comparators (cmps.) involved in these subcircuits are of LM311 type comparators. The subcircuits within the dashed lines colored in green, red and blue are used to increase the number of scrolls in the $x, y$ and $z$ state variable directions, respectively. Since each of these subcircuits includes two
LM311 comparators, a $3 \times 3 \times 3$-grid scroll attractor or 3D-grid scroll attractor can be observed using this circuit. Obviously, by appropriately inserting additional comparators in the corresponding subcircuit, the number of scrolls can be systematically increased in all directions. Also, by appropriately removing these subcircuits, new circuits allowing the observation of any 1D- and 2D-grid scroll attractors can readily be obtained.

For $C_{1}=C_{2}=C_{3}=C, R_{1}=R, R_{2}=$ $R_{4}=R / a, V_{x}=a x, V_{y}=a y, V_{z}=z$ and using the normalized quantity $t_{n}=t / R C$, it can be verified that the circuit realizes the system in Eq. (1). Also, as explained above, the nonlinearities in Eqs. (5), (8) and (9) are realized using the

$\overline{1}^{1}$ Analog Devices [1990] Linear Products Data Book, Norwood, MA, USA. 
subcircuits in red, blue and green, respectively, where the parameters $\theta$ of the core functions, $g_{\theta}(\cdot)$ in Eq. (4) are adjusted through the controlling voltages at the inverting/noninverting inputs of the comparators. It should be noted that all three states are available at the buffered output terminals of the CFOAs, a property which is expected to simplify the realizations of various chaotic communication systems based on the proposed circuit. Also, the fact that all the capacitors are grounded simplifies the IC integration of the circuit. In all the experiments given in this section, CFOAs and LM311 type comparators are supplied under $\pm 15 \mathrm{~V}$ DC and the passive component values are taken as $C_{1}=C_{2}=C_{3}=1 \mathrm{nF}, R_{1}=5.1 \mathrm{k} \Omega$. For all experiments, different values have been assigned to the other passive components. Also, the controlling voltages at the inverting and noninverting inputs of the comparators are taken as adjustable.

First, we have implemented a 5-scroll attractor or 1D-grid scroll attractor from the circuit in Fig. 12. In order to obtain a 5-scroll attractor in the $x$ state variable direction, we have removed the subcircuits in red and blue and added two more comparators to the subcircuit in green. The passive component values are taken as $R_{2}=R_{4}=8 \mathrm{k} \Omega$, $R_{x 1}=R_{x 2}=R_{x 3}=R_{x 4}=70 \mathrm{k} \Omega$. The observed $\left(V_{x}, V_{y}\right)$ trajectory is given in Fig. 13(c). These passive component values correspond to $a=0.64$. Also, by changing the values of the resistors $R_{2}$ and $R_{4}$, the circuit is tested for different values of $a$, and the corresponding results are given in Figs. 13(a)$13(\mathrm{~d})$. These results also verify the dynamic behavior of the circuit with respect to parameter value $a$, which was shown on the simulations of Fig. 4 .

Second, we have implemented $2 \times 2-, 3 \times 2$ - and $3 \times 3$-grid scroll attractors from $2 \mathrm{D}$-grid scroll attractor family. In order to have a $2 \times 2$-grid scroll attractor, we have removed the subcircuit in blue, the comparators $\mathrm{cmp}_{x 2}, \mathrm{cmp}_{y 2}$ and the resistors $R_{x 2}$, $R_{y 2}$, from the circuit in Fig. 12. The passive components values are taken as $R_{2}=R_{4}=9.7 \mathrm{k} \Omega$, $R_{x 1}=37 k \Omega, R_{y 1}=77 k \Omega$. The controlling voltages, i.e. $V_{x 1}, V_{y 1}$ are taken identical. The observed phase space corresponding to the $\left(V_{x}, V_{y}\right)$ trajectory is given in Fig. 14. A $3 \times 2$-grid scroll attractor is realized by adding a comparator, $\operatorname{cmp}_{y 2}$ and a resistor $R_{y 2}$ to the circuit above which was used to

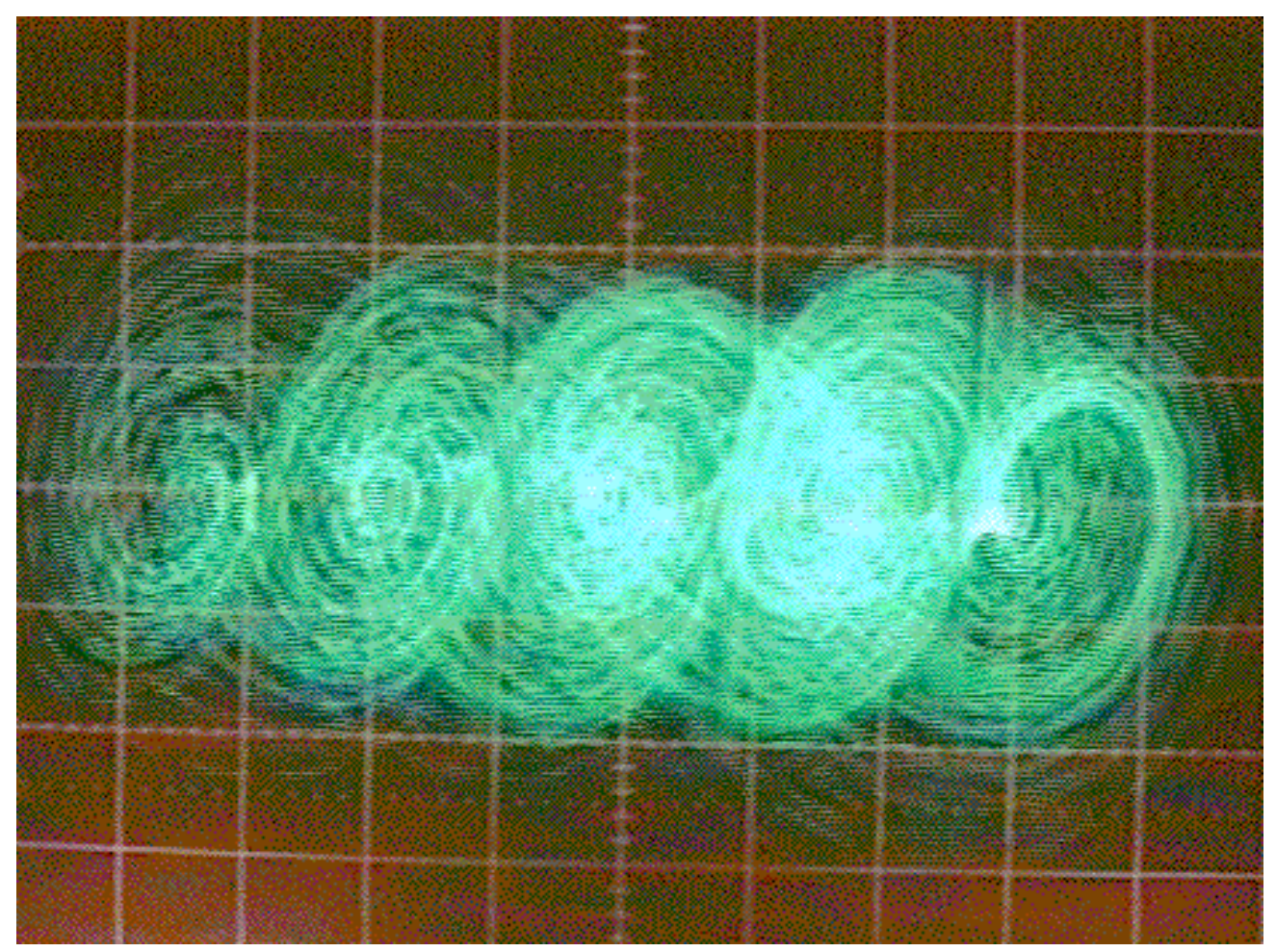

(a)

Fig. 13. 5-scroll attractor from a $1 \mathrm{D}$-grid scroll attractor. Experimental results shown are for $(\mathrm{a}) a=0.26\left(R_{4}=20 \mathrm{k} \Omega\right)$, (b) $a=0.34\left(R_{4}=15 k \Omega\right),\left(\right.$ c) $a=0.64\left(R_{4}=8 k \Omega\right),(\mathrm{d}) a=1\left(R_{4}=5.1 k \Omega\right),\left(V_{x}, V_{y}\right)$ trajectory $x=0.5 \mathrm{~V} /$ div, $y=0.5 \mathrm{~V} / \mathrm{div}$. 


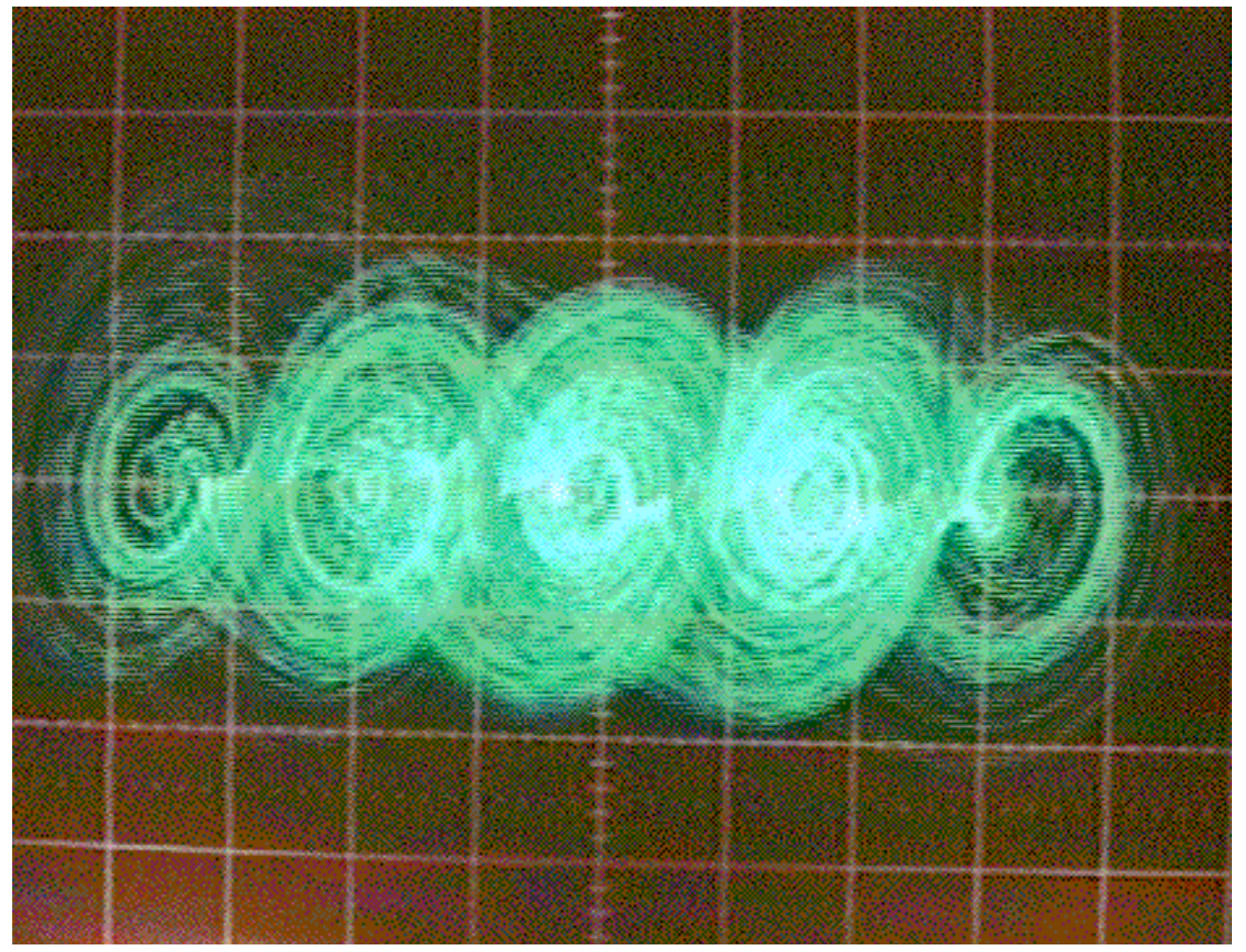

(b)

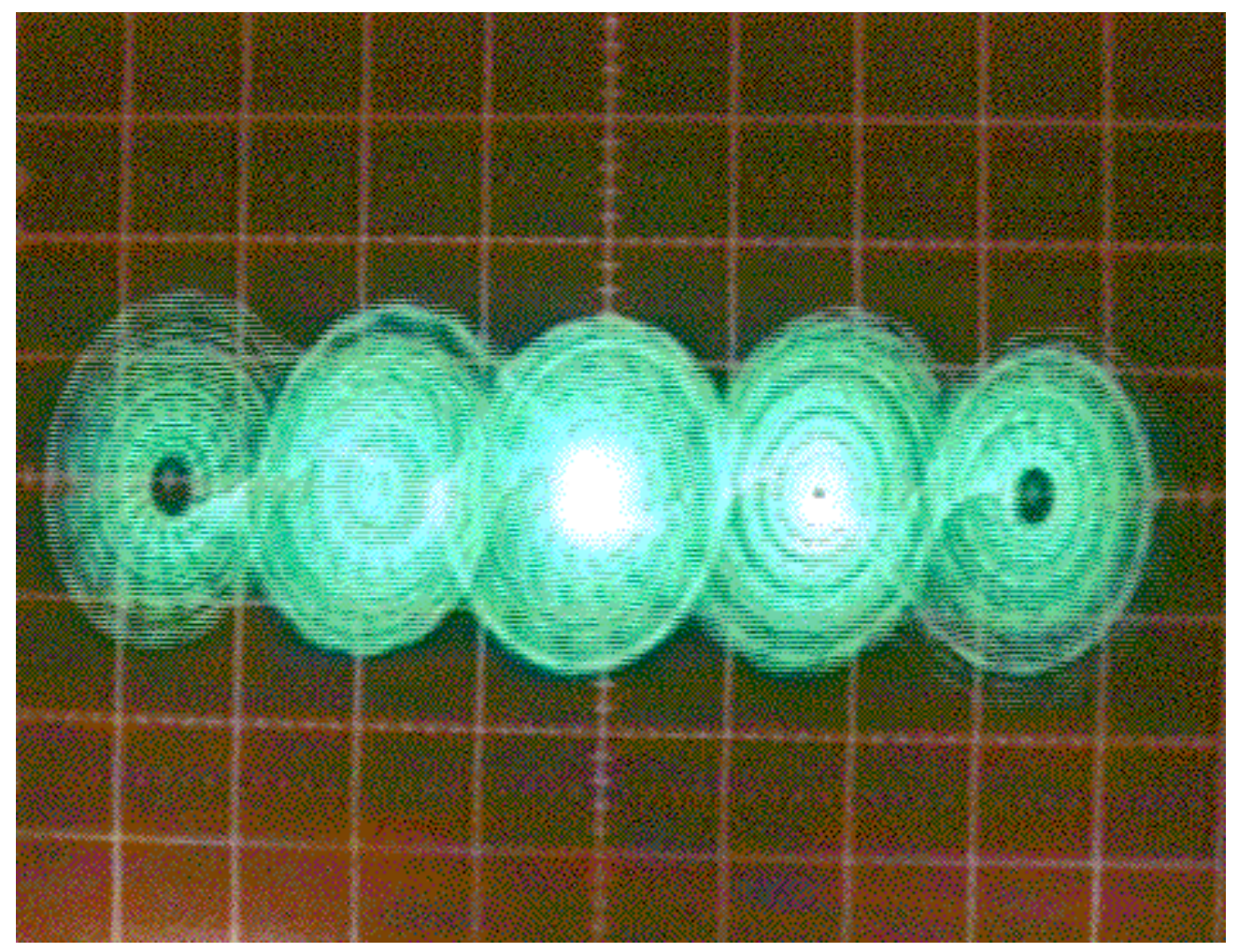

(c)

Fig. 13. (Continued) 


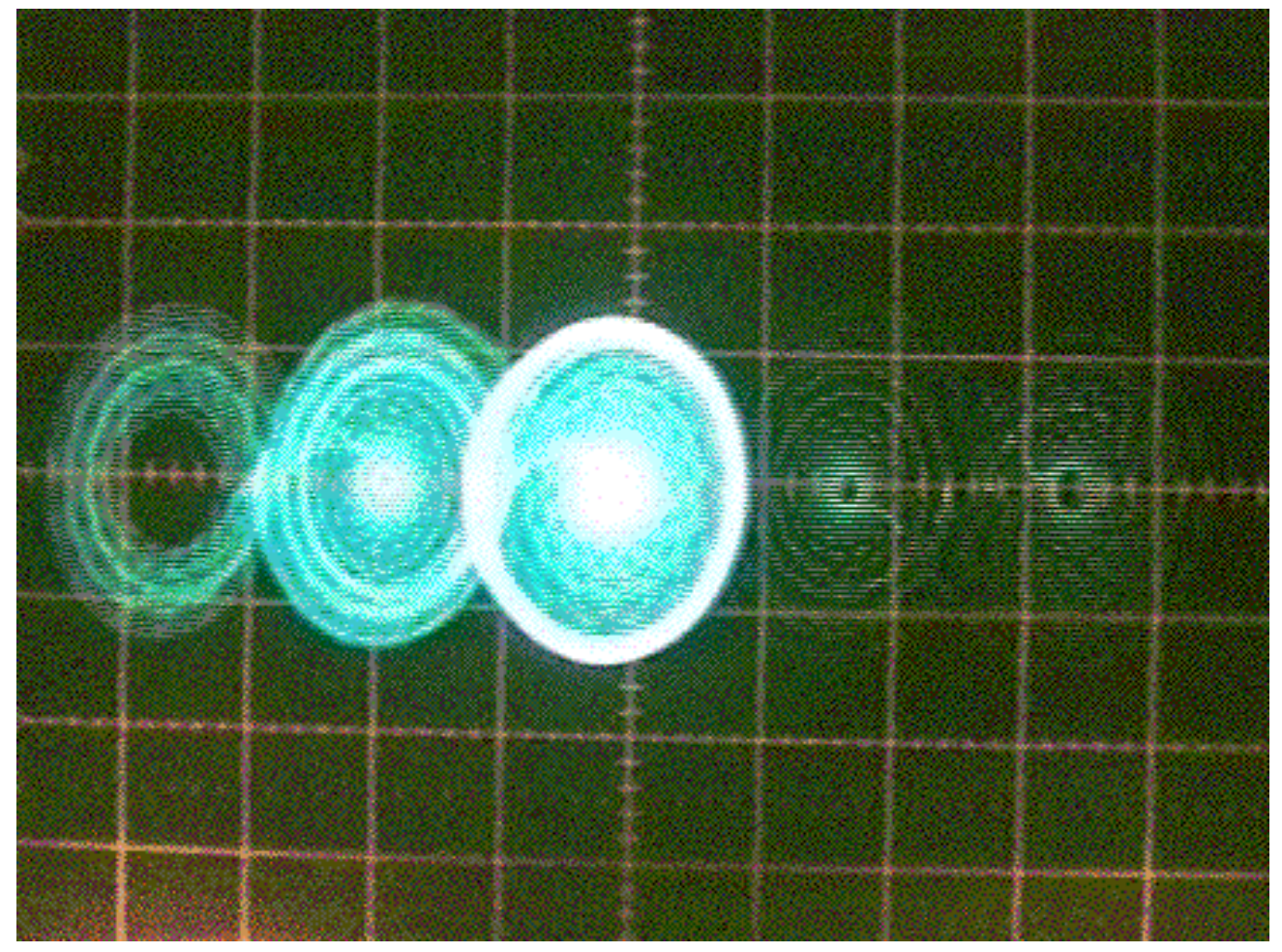

(d)

Fig. 13. (Continued)

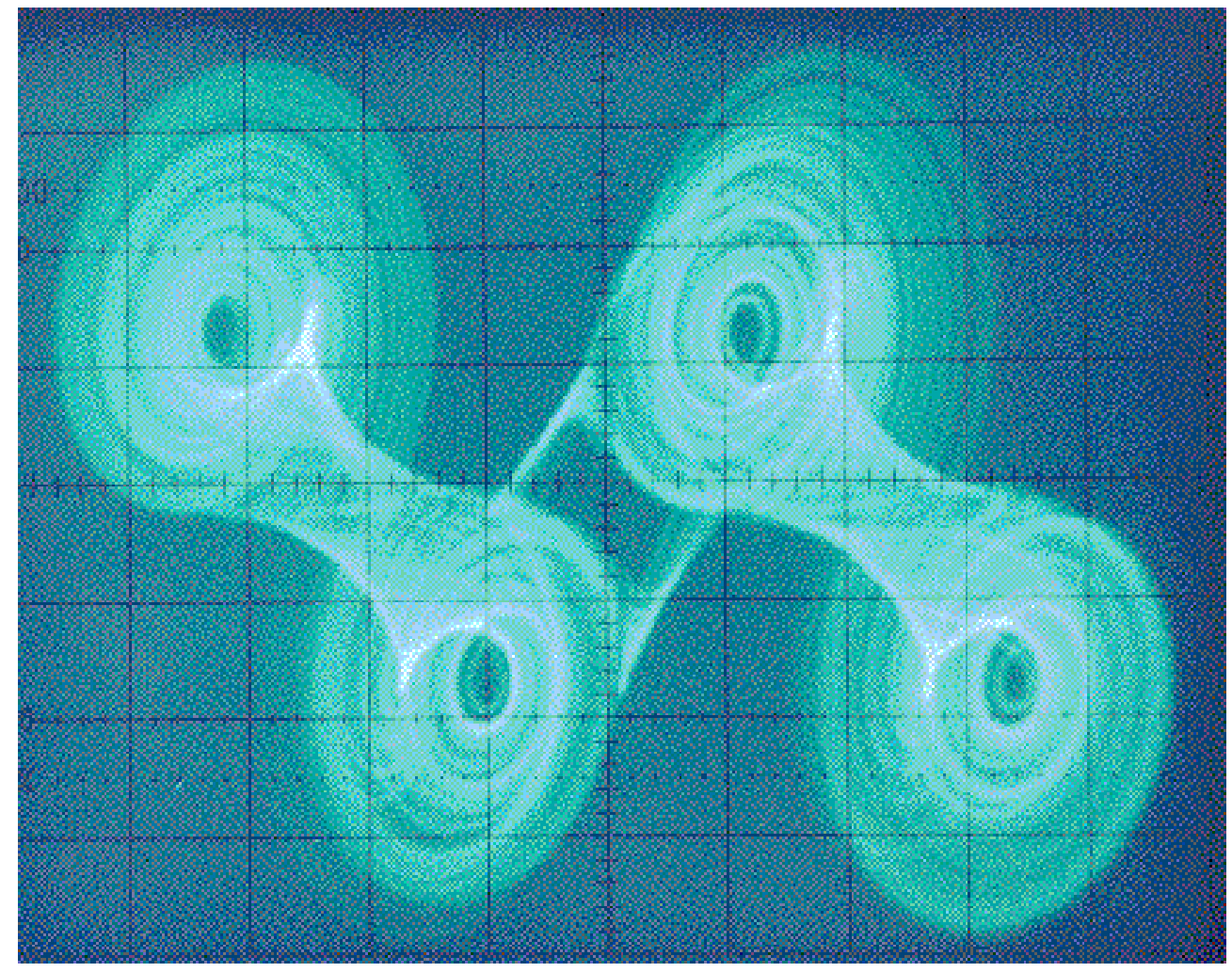

Fig. 14. $2 \times 2$-grid scroll attractor. Experimental result shown is $\left(V_{x}, V_{y}\right)$ trajectory. $x=0.5 \mathrm{~V} /$ div, $y=0.5 \mathrm{~V} /$ div. 


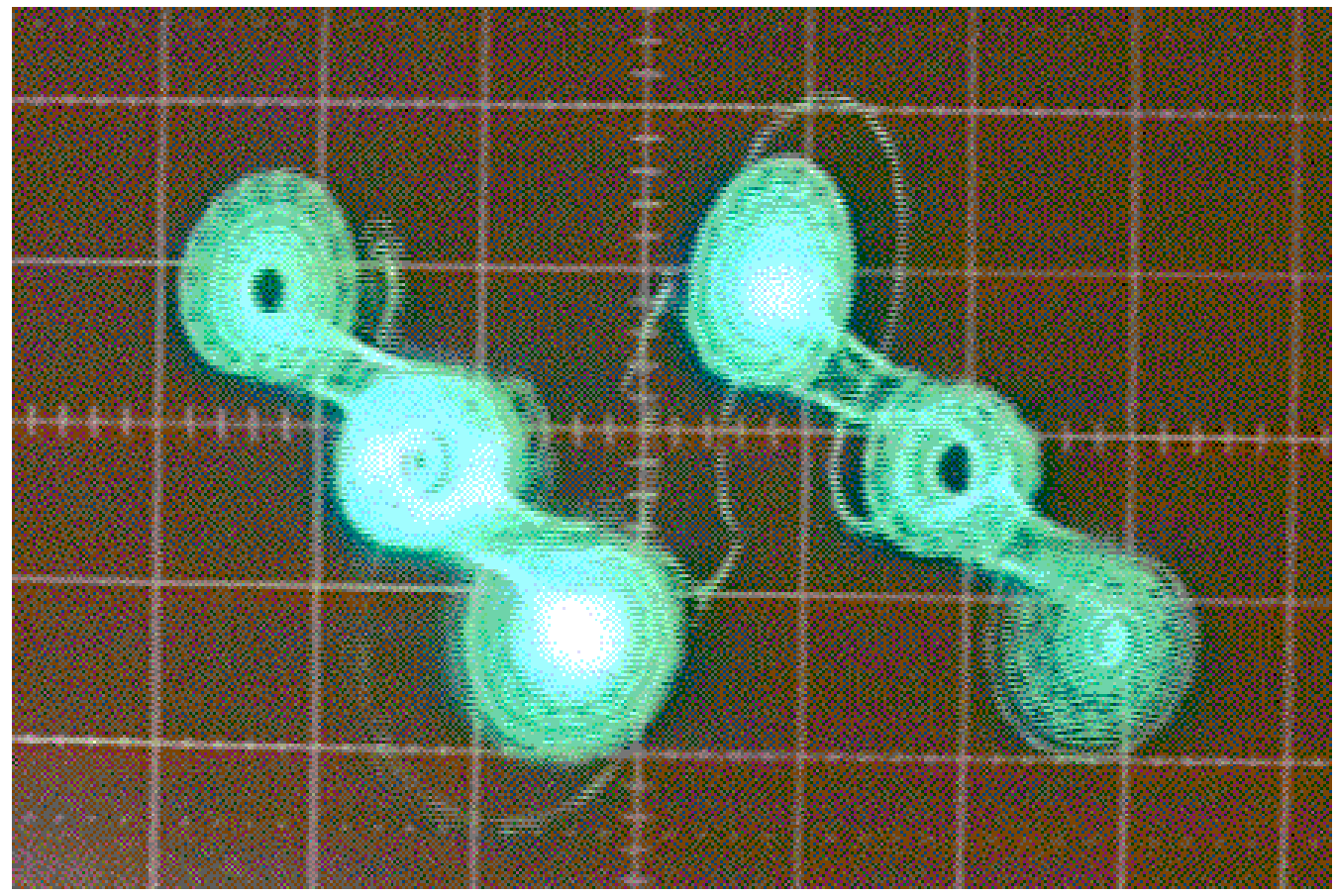

Fig. 15. $2 \times 3$-grid scroll attractor. Experimental result shown is $\left(V_{x}, V_{y}\right)$ trajectory. $x=1 \mathrm{~V} / \operatorname{div}, y=0.5 \mathrm{~V} /$ div.

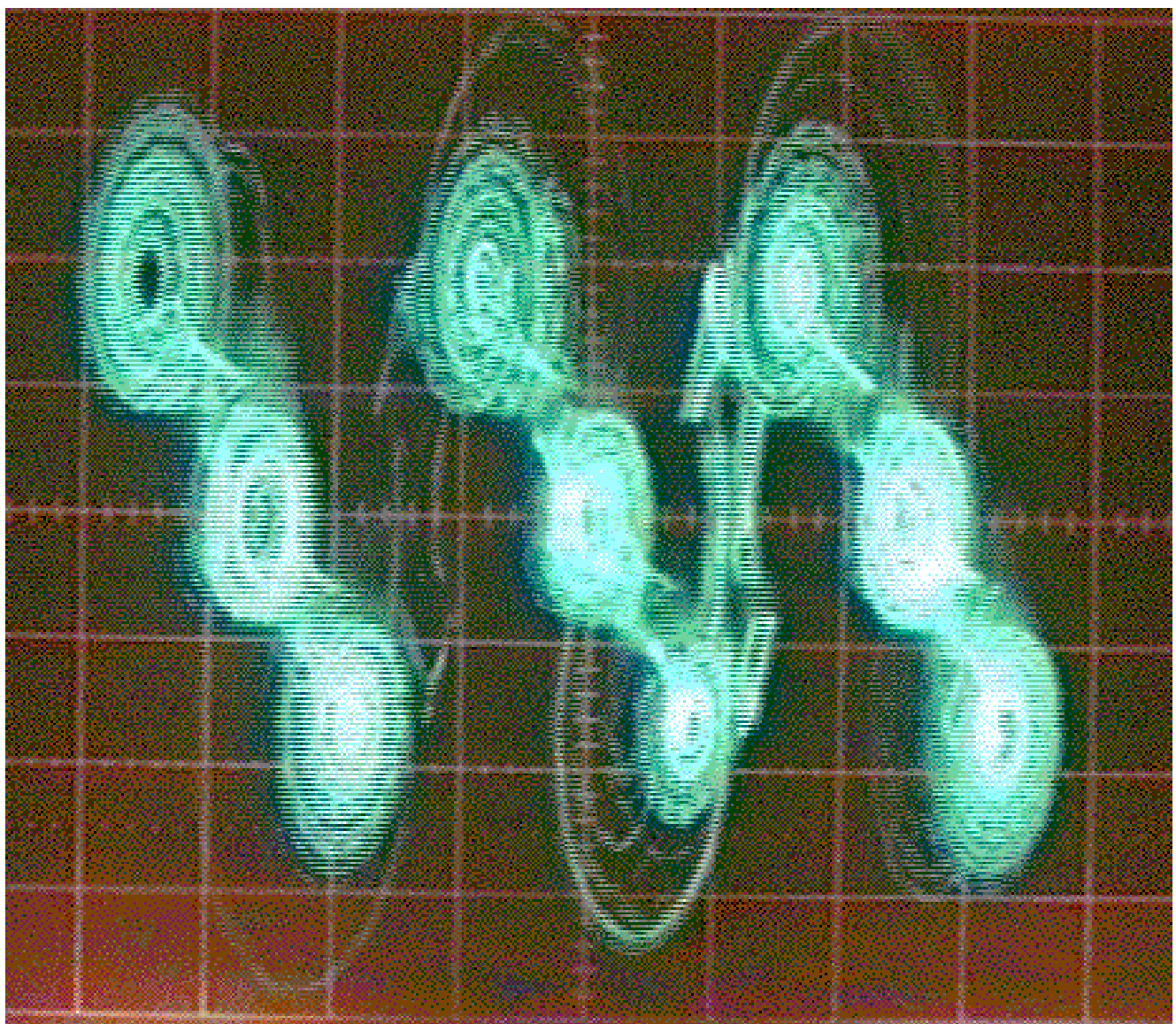

Fig. 16. $3 \times 3$-grid scroll attractor. Experimental result shown in $\left(V_{x}, V_{y}\right)$ trajectory. $x=1 \mathrm{~V} /$ div, $y=0.5 \mathrm{~V} /$ div. 


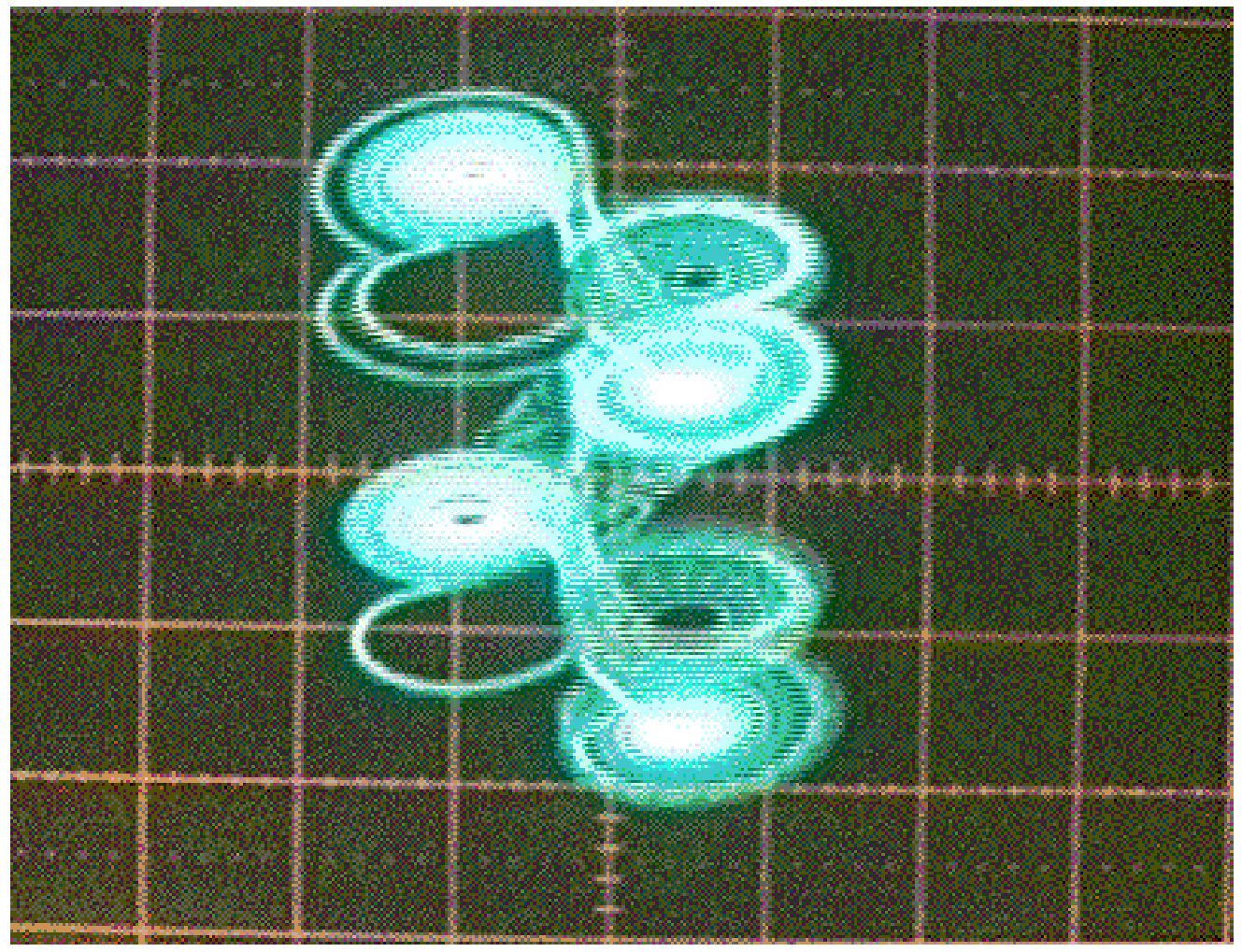

(a)

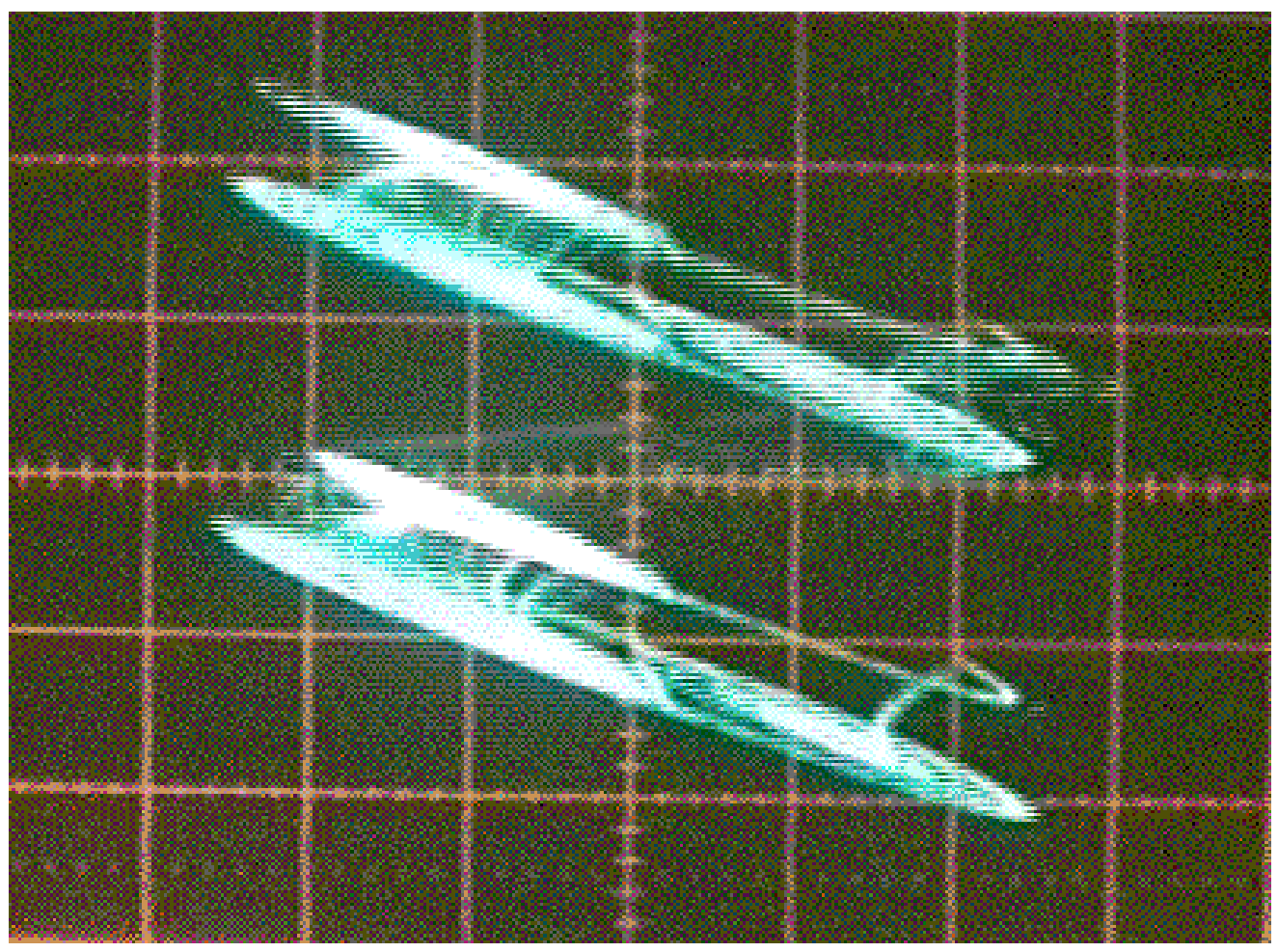

(b)

Fig. 17. $2 \times 2 \times 2$-grid scroll attractor. Experimental results shown are (a) $\left(V_{y}, V_{x}\right)$ trajectory. $x=1 \mathrm{~V} /$ div, $y=2 \mathrm{~V} /$ div, (b) $\left(V_{z}, V_{x}\right)$ trajectory. $x=1 \mathrm{~V} / \operatorname{div}, y=2 \mathrm{~V} / \operatorname{div}$, (c) $\left(V_{y}, V_{z}\right)$ trajectory. $x=1 \mathrm{~V} / \operatorname{div}, y=2 \mathrm{~V} /$ div. 


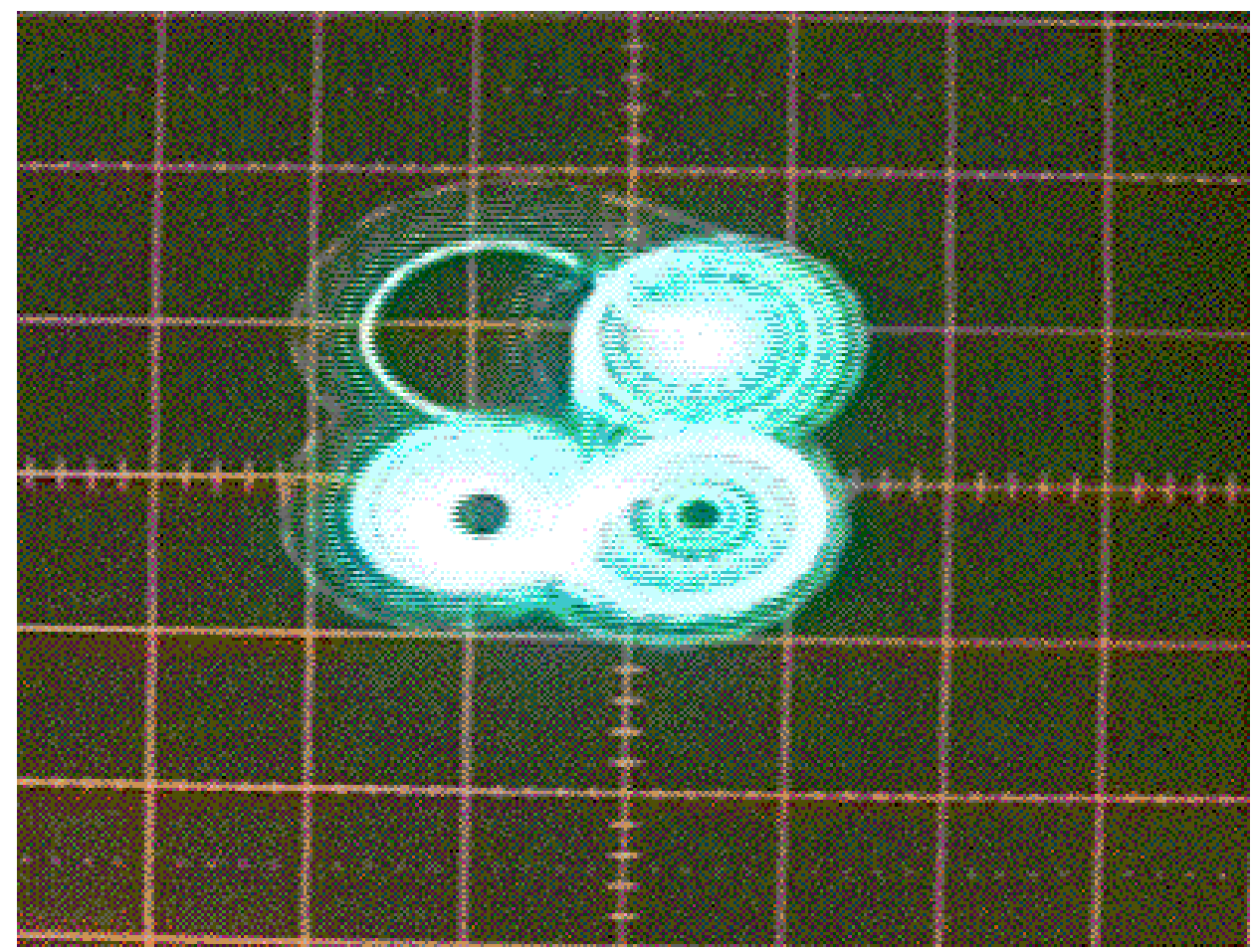

(c)

Fig. 17. (Continued)

realize a $2 \times 2$-grid scroll attractor. The passive component values are taken as $R_{2}=R_{4}=11 \mathrm{k} \Omega$, $R_{x 1}=27 \mathrm{k} \Omega, R_{y 1}=88 \mathrm{k} \Omega, R_{y 2}=75 \mathrm{k} \Omega$. The controlling voltages $V_{x 1}, V_{y 1}$ are taken to be identical. The observed $\left(V_{x}, V_{y}\right)$ trajectory shown in Fig. 15 verifies the theory. As a final example of the 2D-grid attractor family, we have realized a $3 \times 3$-grid attractor by increasing the number of comparators in the subcircuit in green by one. In this case, the passive component values are chosen as $R_{2}=R_{4}=12 \mathrm{k} \Omega, R_{x 1}=28 \mathrm{k} \Omega, R_{x 2}=30 \mathrm{k} \Omega$, $R_{y 1}=90 \mathrm{k} \Omega, R_{y 2}=80 \mathrm{k} \Omega$. Again, the measured $\left(V_{x}, V_{y}\right)$ trajectory is given in Fig. 16 .

Finally, a $2 \times 2 \times 2$-grid attractor from the $3 \mathrm{D}$-grid attractor family is realized using the circuit in Fig. 12. In order to have a $2 \times 2 \times 2$-grid scroll attractor with $M_{y}=0, N_{y}=1, M_{z}=0$, $N_{z}=1$ and $k=2$, we have removed the comparators $\mathrm{cmp}_{x 2}, \mathrm{cmp}_{y 2}$ and $\mathrm{cmp}_{z 2}$ in the subcircuits within the dashed lines and the passive component values are taken as $R_{2}=R_{4}=8.3 \mathrm{k} \Omega, R_{x 1}=19 \mathrm{k} \Omega$, $R_{y 1}=47 \mathrm{k} \Omega, R_{z 1}=50 \mathrm{k} \Omega$. The controlling voltages at the comparator inputs are taken as identical. The experimental results corresponding to the $\left(V_{y}, V_{x}\right),\left(V_{z}, V_{x}\right)$ and $\left(V_{y}, V_{z}\right)$ trajectories are given in Fig. 17.

\section{Conclusions}

Since the introduction of Chua's circuit, several multi scroll-based chaotic attractors have been presented in the literature. However, up till now it was not possible to generate the scrolls in different directions. In this paper, we have shown that it is possible to create strange attractors whose scrolls can be located in any state variable direction. Following the ideas outlined in this paper, the design of new attractors depends on the designer's imagination, as the presented attractors are just samples derived from the new proposed family which might be further extended in the future. The proposed system presented in this work is expected to yield new chaotic signal generators which can be useful in many chaos-based applications.

\section{Acknowledgments}

This research work was carried out at the ESAT laboratory and the Interdisciplinary Center of Neural Networks ICNN of the Katholieke Universiteit Leuven, in the framework of the Belgian Programme on Interuniversity Poles of Attraction, initiated by the Belgian State, Prime Minister's Office for 
Science, Technology and Culture (IUAP P4-02), the Concerted Action Project MEFISTO of the Flemish Community, the FWO project Collective Behavior and Optimization: an Interdisciplinary Approach and ESPRIT IV 27077 (DICTAM). Johan Suykens is a postdoctoral researcher with the National Fund for Scientific Research FWO - Flanders.

\section{References}

Arena, P., Baglio, S., Fortuna, L. \& Manganaro, G. [1996] "Generation of $n$-double scrolls via cellular neural networks," Int. J. Circuit Th. Appl. 24, 241-252.

Chua, L. O., Komuro, M. \& Matsumoto, T. [1986] "The double scroll family," IEEE Trans. Circuits Syst. I 33(11), 1072-1118.

Chua, L. O. \& Roska, T. [1993] "The CNN paradigm," IEEE Trans. Circuits Syst. I 40(3), 147-156.

Chua, L. O. [1994] "Chua's circuit 10 years later," Int. J. Circuit Th. Appl. 22, 279-305.

Elwakil, A. S. \& Kennedy, M. P. [2000] "Improved implementation of Chua's chaotic oscillator using current feedback opamp," IEEE Trans. Circuits Syst. I 47(1), 76-79.

Elwakil, A. S., Salama, K. N. \& Kennedy, M. P. [2000] "A system for chaos generation and its implementation in monolithic form," Proc. IEEE Int. Symp. Circuits and Systems (ISCAS 2000) (V), pp. 217-220.

Fabre, A. [1993] "Insensitive voltage-mode and currentmode filters from commercially available transimpedance op-amps," IEE Proc. G 140(5), 319-321.

Hasler, M. [1994] "Synchronization principles and applications," Circuit $\mathcal{E}$ Systems: Tutorials IEEE-ISCAS '94, pp. 314-326.

Kapitaniak, T. \& Chua, L. O. [1994] "Hyperchaotic attractors of unidirectionally-coupled Chua's circuit," Int. J. Bifurcation and Chaos 4(2), 477-482.

Kataoka, M. \& Saito, T. [2000] "A 4-D chaotic oscillator with a hysteresis 2-Port VCCS," Proc. IEEE Int. Symp. Circuits and Systems (ISCAS 2000) (V), pp. $418-421$.

Khalil, H. K. [1993] Nonlinear Systems (Macmillan Publishing Company, NY).

Kolumban, G., Kennedy, M. P. \& Chua, L. O. [1998] "The role of synchronization in digital communica- tions using chaos-Part II: Chaotic modulation of digital communications," IEEE Trans. Circuits Syst. I 45(11), 1129-1140.

Madan, R. N. [1993] Chua's Circuit: A Paradigm for Chaos (World Scientific, Singapore).

Saito, T. [1990] "An approach toward higher dimensional hysteresis chaos generators," IEEE Trans. Circuits Syst. I 37(3), 399-409.

Senani, R. \& Gupta, S. S. [1998] "Implementation of Chua's chaotic circuits using current feedback opamps," Electron. Lett. 34(9), 829-830.

Suykens, J. A. K. \& Vandewalle, J. [1993] "Generation of $n$-double scrolls $(n=1,2,3,4, \ldots), "$ IEEE Trans. Circuits Syst. I 40(11), 861-867.

Suykens, J. A. K. \& Chua, L. O. [1997] " $n$-Double scroll hypercubes in 1-D CNNs," Int. J. Bifurcation and Chaos 7(8), 1873-1885.

Suykens, J. A. K., Huang, A. \& Chua, L. O. [1997] "A family of $n$-scroll attractors from a generalized Chua's circuit," Archiv für Elektronik und Ubertragungstechnik (Int. J. Electronics and Communications) 51(3), 131-138.

Suykens, J. A. K., Curran, P. F. \& Chua, L. O. [1999] "Robust synthesis for master-slave synchronization of Lur'e Systems," IEEE Trans. Circuits Syst. I 46(7), 841-850.

Toumazou, C., Lidgey, F. J. \& Haigh, D. Q. [1990] Analog IC Design: The Current Mode Approach (Peter Peregrinus, London).

Toumazou, C. \& Lidgey, F. J. [1994] "Current feedback op-amps: A blessing in disguise?" IEEE Circuits Devices Mag. 10(1), 43-47.

Vidyasagar, M. [1993] Nonlinear Systems Analysis (Prentice-Hall).

Yalçın, M. E., Suykens, J. A. K. \& Vandewalle, J. [2000a] "Experimental confirmation of 3- and 5-scroll attractors from a generalized Chua's circuit," IEEE Trans. Circuits Syst. I 47(3), 425-429.

Yalçın, M. E., Suykens, J. A. K. \& Vandewalle, J. [2000b] "Hyperchaotic $n$-scroll attractors," Proc. IEEE Workshop on Nonlinear Dynamics of Electronic Systems (NDES 2000), pp. 25-28.

Yalçın, M. E., Özoguz, S., Suykens, J. A. K. \& Vandewalle, J. [2000c] " $n$-Scroll chaos generators: A simple circuit model," Electron. Lett. 37(3), 147-148. 